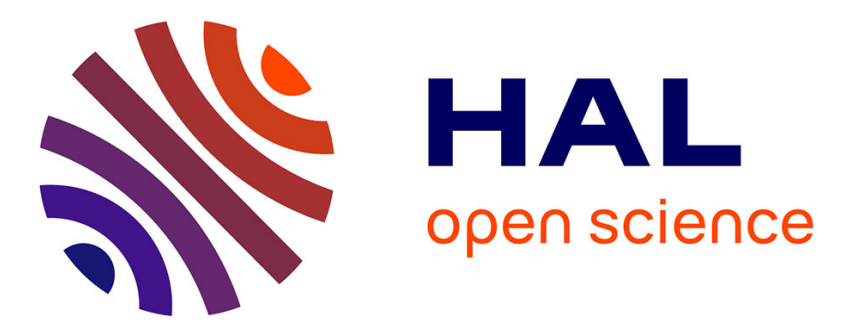

\title{
Generalized Homogenization of Linear Controllers: Theory and Experiment
}

\author{
Siyuan Wang, Andrey Polyakov, Gang Zheng
}

\section{To cite this version:}

Siyuan Wang, Andrey Polyakov, Gang Zheng. Generalized Homogenization of Linear Controllers: Theory and Experiment. International Journal of Robust and Nonlinear Control, In press, 10.1002/rnc.5112 . hal-02877410

HAL Id: hal-02877410

https://hal.inria.fr/hal-02877410

Submitted on 22 Jun 2020

HAL is a multi-disciplinary open access archive for the deposit and dissemination of scientific research documents, whether they are published or not. The documents may come from teaching and research institutions in France or abroad, or from public or private research centers.
L'archive ouverte pluridisciplinaire HAL, est destinée au dépôt et à la diffusion de documents scientifiques de niveau recherche, publiés ou non, émanant des établissements d'enseignement et de recherche français ou étrangers, des laboratoires publics ou privés. 


\title{
Generalized Homogenization of Linear Controllers: Theory and Experiment
}

\author{
Siyuan Wang, Andrey Polyakov, Gang Zheng \\ Inria Lille-Nord Europe, France
}

June 22, 2020

\begin{abstract}
A procedure for an "upgrade" of a linear PID controller to a non-linear homogeneous one is developed and verified by real experiments with quadrotor. The controller design is based on a generalized homogeneity (dilation symmetry) of the system. Its parameters are obtained from the gains of linear controller. The issues of digital implementation of the proposed controller are discussed. Finally, the stability and robustness properties of the homogeneous PID controller are validated on the quadrotor QDrone platform of Quanser ${ }^{T M}$.
\end{abstract}

\section{Introduction}

\subsection{State of the art}

Homogeneity is a kind of symmetry when an object (a function, a set, etc) remains invariant with respect to a class of transformations called dilations. For example, a symmetry of a function $f$ with respect to the uniform dilation of its argument $x \rightarrow e^{s} x$, where $s \in \mathbb{R}$ is the scaling parameter, is known as standard homogeneity. Any standard homogeneous function $f$ is characterized as follows $f\left(e^{s} x\right)=e^{\nu s} f(x), \forall s \in$ $\mathbb{R}, \forall x \in \mathbb{R}^{n}$, where a constant $\nu \in \mathbb{R}$ is called homogeneity degree. The standard homogeneity is introduced by means of the uniform dilation. It is clear that if we change the dilation rule then another type of homogeneity can also be defined. The weighted dilation (introduced by V.I. Zubov in 1958, [1]) of the vector $x=\left(x_{1}, x_{2}, \ldots, x_{n}\right)^{\top} \in \mathbb{R}^{n}$ is the simplest example of the so-called generalized (non-uniform) dilation: $\left(x_{1}, x_{2}, \ldots, x_{n}\right) \rightarrow\left(e^{r_{1} s} x_{1}, e^{r_{2} s} x_{2}, \ldots, e^{r_{n} s} x_{n}\right)$, where $s \in \mathbb{R}$ as before is the scaling parameter and the positive numbers $r_{1}, r_{2}, \ldots, r_{n}$ are the weights specifying dilation rates along different coordinates. In this paper we deal mainly with the linear geometric dilation given by $x \rightarrow e^{G_{\mathbf{d}} s} x$, where $x \in \mathbb{R}^{n}, s \in \mathbb{R}$ and $G_{\mathbf{d}} \in \mathbb{R}^{n \times n}$ is an anti-Hurwitz matrix.

All linear and a lot of essentially nonlinear models of mathematical physics are homogeneous (symmetric) in a generalized sense [2], [3]. Homogeneous models are utilized as local approximations of control systems [1], [4] if, for example, linearization is too conservative, non-informative or simply impossible. Many methods of both linear and non-linear control theory can be applied for analysis and design of homogeneous control systems [5], [6], [7].

Generalized homogeneous control laws appear as solutions of some classical control problems such as a minimum-time feedback control for the chain of integrators [8]. Most of the high-order sliding mode control and estimation algorithms are homogeneous in a generalized sense [9]. Homogeneity allows time constraints in control systems to be fulfilled easily by means of a proper tuning of the so-called homogeneity degree [10]. Similarly to the linear case, stability of a homogeneous system implies its robustness (input-to-state stability) with respect to some classes of parametric uncertainties and exogenous perturbations [4], [11].

Many different homogeneous controller are designed for linear plants (basically, for a chain of integrators), see e.g. [4], [12], [13], [9], among others. Usually, an existence of homogeneous controller of a certain form has been proven, however a proper tuning of control parameters also needs to be studied. In addition, it is 
not clear if, in practice, a homogeneous controller could have a better performance than a well-tuned linear regulator.

\subsection{Homogeneity vs linearity}

Quality of any control system is estimated by many quantitative indexes (see e.g. [14], [15], [16]), which reflect control precision, energetic effectiveness, robustness of the closed-loop system with respect to disturbances, etc. From mathematical point of view, the design of a "good" control law is a multi-objective optimization problem. The mentioned criteria frequently contradict to each other, e.g. a time optimal feedback control could not be energetically optimal but it may be efficient for disturbance rejection [8]. In practice, an adjustment of a guaranteed (small enough) convergence time can be considered instead of minimum time control problem, and an exact convergence of systems states to a set-point is relaxed to a convergence into a sufficiently small neighborhood of this set-point.

A well-tuned linear controller, such as PID (Proportional-Integral-Differential) algorithm, guarantees a good enough control quality in many practical cases [14]. However, the further improvement of control performance using the same linear strategy looks impossible. Being a certain relaxation of linearity, the homogeneity could provide additional tools for improving control quality. In this context, it is worth to know if there exist some theoretical features of homogeneous systems, which may be useful (in practice) for a design of an advanced control system.

\section{Finite-time and fixed-time stabilization}

Finite-time and fixed-time stability are a rather interesting theoretical features of homogeneous systems [13], [5], [17]. For example, if an asymptotically stable system is homogeneous of positive degree at infinity and homogeneous of negative degree at the origin then any its trajectory reaches the origin (a set point) in a fixed time independently of the initial condition [4]. This idea can be illustrated on the simplest scalar example

$$
\dot{x}(t)=u(t), \quad t>0, \quad x(0)=x_{0},
$$

where $x(t) \in \mathbb{R}$ is the state variable and $u(t) \in \mathbb{R}$ is the control signal. The control aim is to stabilize this system at the origin such that the condition $|u(x)| \leq 1$ must be fulfilled for $|x| \leq 1$.

- The classical approach gives the standard linear proportional feedback algorithm

$$
u_{\text {lin }}(x)=-x
$$

which guarantees asymptotic (in fact, exponential) convergence to the origin of any trajectory of the closed-loop system:

$$
|x(t)|=e^{-t}\left|x_{0}\right|
$$

- The globally homogeneous feedback of the form

$$
u_{f t}(x)=-\sqrt{|x|} \operatorname{sign}[x] .
$$

stabilizes the system at the origin in a finite-time:

$$
x(t)=0, \quad \text { for } t \geq T\left(x_{0}\right) .
$$

The corresponding convergence time $T$ depends on the initial condition $x(0)=x_{0}$, in particular, $T\left(x_{0}\right)=2 \sqrt{\left|x_{0}\right|}$ for the considered control law.

- The fixed-time stabilizing controller is locally homogeneous and has the form:

$$
u_{f x t}(x)=-\frac{1}{2}\left(|x|^{1 / 2}+|x|^{3 / 2}\right) \operatorname{sign}[x] .
$$

It guarantees a global uniform boundedness of the settling time, namely,

$$
x(t)=0, \quad t \geq 2 \pi
$$

for the considered control law. 


\section{Robustness issues}

Let us consider the simplest stabilization problem

$$
\dot{x}=\lambda x+u
$$

where $x \in \mathbb{R}$ is the system state, $\lambda>0$ is an unknown constant parameter and $u \in \mathbb{R}$ is a state feedback to be designed. Since $\lambda$ is unknown then any static linear feedback $u=-k x$ cannot guarantee a priori a boundedness of system trajectories. However, the homogeneous feedback

$$
u=-k x^{2} \operatorname{sign}(x), \quad k>0
$$

always ensures practical stabilization of the system independently of the parameter $\lambda$. Indeed, estimating the derivative of the energy $V=x^{2}$ of the system along the trajectories we derive

$$
\frac{d}{d t} x^{2} \leq 2 \lambda x^{2}-2 k|x|^{3}<0 \quad \text { for } \quad|x|>\lambda / k .
$$

This means boundedness of system trajectories and convergence to a zone:

$$
\limsup _{t \rightarrow+\infty}|x(t)| \leq \frac{\lambda}{k}
$$

Therefore, the homogeneous control system is robust with respect to larger class of uncertainties than the linear control system.

\section{Elimination of an unbounded "peaking" effect}

Finite-time and fixed-time stability is an interesting theoretical feature of homogeneous systems. However, a controllable linear system can be stabilized in a small neighborhood of a set-point even by means of a static linear feedback. A time of convergence of trajectories from the unit ball into this neighborhood can be prescribed in advance by means of an appropriate tuning of the feedback gain. Such a stabilization is sufficient for many practical problems. The reasonable question in this case: is there any advantage of a homogeneous controller comparing with a linear feedback?

Let us consider the control system

$$
\dot{x}=A x+b u(x), \quad t>0, \quad A=\left(\begin{array}{ccccc}
0 & 1 & 0 & \cdots & 0 \\
0 & 0 & 1 & \cdots & 0 \\
\cdots & 0 & \cdots & \cdots & 0 \\
0 & 0 & 0 & \cdots & \cdots \\
0 & 0 & 0 & \cdots & 0
\end{array}\right), \quad B=\left(\begin{array}{c}
0 \\
0 \\
\vdots \\
0 \\
1
\end{array}\right)
$$

where $x=\left(x_{1}, x_{2}, \ldots, x_{n}\right)^{\top}$ is the state vector and $u: \mathbb{R}^{n} \rightarrow \mathbb{R}$ is the feedback control.

Initial conditions of the latter system are assumed to be bounded as follows

$$
\|x(0)\| \leq 1 .
$$

The control aim is to stabilize the state vector $x$ of the system into a ball of a small radius $\varepsilon>0$ in a prescribed time $T>0$, i.e.

$$
\|x(t)\| \leq \varepsilon, \quad \forall t \geq T .
$$

Let us consider the static linear feedback

$$
u_{\ell}(x)=k x, \quad k=\left(k_{1}, k_{2}, \ldots, k_{n}\right) .
$$

The eigenvalues $\left\{\lambda_{1}, \ldots, \lambda_{n}\right\}$ of the closed-loop linear system

$$
\dot{x}=(A+b k) x
$$

can be placed in any given set of the complex plane $\mathbb{C}$ by choosing the vector $k$. Therefore, it is possible to obtain a closed-loop system with an arbitrary fast damping speed, i.e.

$$
\forall \varepsilon>0, \quad \exists k \in \mathbb{R}^{1 \times n}: \sup _{\left\|x_{0}\right\|=1}\|x(t)\|<\varepsilon, \quad t>T .
$$


Indeed, the trajectories of this system converge to the origin exponentially fast

$$
\|x(t)\| \leq C e^{-\sigma t}, t>0
$$

where the constant $C \geq 1$ depends on $\lambda_{i}, i=1,2, . ., n$ and $\Re\left(\lambda_{i}\right)<-\sigma$. Hence, smaller $\varepsilon>0$ larger $\sigma>0$ has to be assigned to solve the control problem, i.e. $\sigma \rightarrow+\infty$ as $\varepsilon \rightarrow 0$ provided that $T$ is fixed. Therefore, we conclude that the linear state feedback is, indeed, a possible solution of the considered stabilization problem for any fixed $\varepsilon>0$.

However, the trajectories of the closed-loop linear system with fast decays have large deviations from the origin during the initial phase of the stabilization. This phenomenon is called the "peaking" effect and the large deviation is referred to as an "overshoot" (see e.g. [18] for more details). In particular, it is shown [19] that there exists $\gamma>0$ independent of $\lambda_{i}$ such that

$$
\sup _{0 \leq t \leq \sigma^{-1}} \sup _{\|x(0)\|=1}\|x(t)\| \geq \gamma \sigma^{n-1} .
$$

For $n>1$ the linear closed-loop system has infinite "overshoot" as $\varepsilon \rightarrow 0$ :

$$
\sup _{0 \leq t \leq T} \sup _{\|x(0)\|=1}\|x(t)\| \rightarrow+\infty \quad \text { as } \quad \varepsilon \rightarrow 0 .
$$

This means that for sufficiently small $\varepsilon>0$ the "overshoot" may be so huge that physical (practical) restrictions of the system states would not allow it. The static linear control needs to be somehow modified to overcome this difficulty. The simplest way is to use the input saturation, which, in fact, anyway must be taken into account in practice. However, in this case it is not clear if the saturated feedback would solve the considered stabilization problem with the prescribed time $T>0$ even if saturation would not destroy stability of the system trajectories initiated in the unit ball.

The infinite "peaking" effect could also be eliminated by means of a transformation of the linear controller to a homogeneous one. Indeed, let us consider the following feedback law

$$
u_{h}(x)=\tilde{k} \mathbf{d}\left(-\ln \|x\|_{\mathbf{d}}\right) x,
$$

where $\mathbf{d}$ is the weighted dilation

$$
\mathbf{d}(s)=\left(\begin{array}{cccc}
e^{n s} & 0 & \cdots & 0 \\
0 & e^{(n-1)} & \cdots & 0 \\
\cdots & \ldots & \cdots & \cdots \\
0 & 0 & \cdots & e^{s}
\end{array}\right), \quad s \in \mathbb{R}
$$

and $\|\cdot\|_{\mathbf{d}}: \mathbb{R}^{n} \rightarrow(0,+\infty)$ is the so-called canonical homogeneous norm studied in [20]. Since $\|\mathbf{d}(s) x\|_{\mathbf{d}}=$ $e^{s}\|x\|_{\mathbf{d}}$ then the vector field $f$ given by

$$
f(x):=A x+b u_{h}(x)
$$

is weighted homogeneous of degree -1 , i.e. $f(\mathbf{d}(s) x)=e^{-s} \mathbf{d}(s) f(x)$.

Below we show that the vector $\tilde{k}=\left(\tilde{k}_{1}, \tilde{k}_{2}, \ldots, \tilde{k}_{n}\right)^{\top}$ can be selected to guarantee

$$
\sup _{\|x(0)\|=1}\|x(t)\|=0, \quad t \geq T
$$

for any fixed $T>0$. In addition, the feedback law $u_{h}$ is globally bounded:

$$
\sup _{x \in \mathbb{R}^{n}}|u(x)| \leq M<+\infty
$$

where $M$ depends on $T$ as follows: smaller $T$ implies larger $M$. The homogeneous control stabilizes the considered system globally and in a finite time. It solves the stabilization problem considered above independently of $\varepsilon>0$. Due to global boundedness of the controller it does not have the unbounded "peaking" effect discovered for the linear system as $\varepsilon \rightarrow 0$.

The simulation results for the linear controller $u(x)=k x, k=(-100-20)$ and the homogeneous controller $u_{h}(x)=\tilde{k}\left(\begin{array}{cc}\|x\|_{\mathbf{d}}^{-2} & 0 \\ 0 & \|x\|_{\mathbf{d}}^{-1}\end{array}\right) x, \tilde{k}=(-4.1721-2.8718)$ are depicted in Figure 1. Initial conditions $x(0)$ for the 
numerical simulations has been taken from the unit sphere. In both case, trajectories of the closed-loop system converge to the origin. The homogeneous controller provides the (theoretically) exact stabilization of any solution of the closed-loop system with $\|x(0)\| \leq 1$ in the time $T=1$, i.e. $x(t)=0$ for all $t \geq 1$ and for all $\|x(0)\| \leq 1$. The linear controller just guarantees $\|x(t)\| \leq \varepsilon=0.005$ for $t \geq 1$. Even in this case the "overshoot" of the homogeneous controller is twice smaller. The "overshoot" of the linear controller increases drastically for smaller $\varepsilon$.
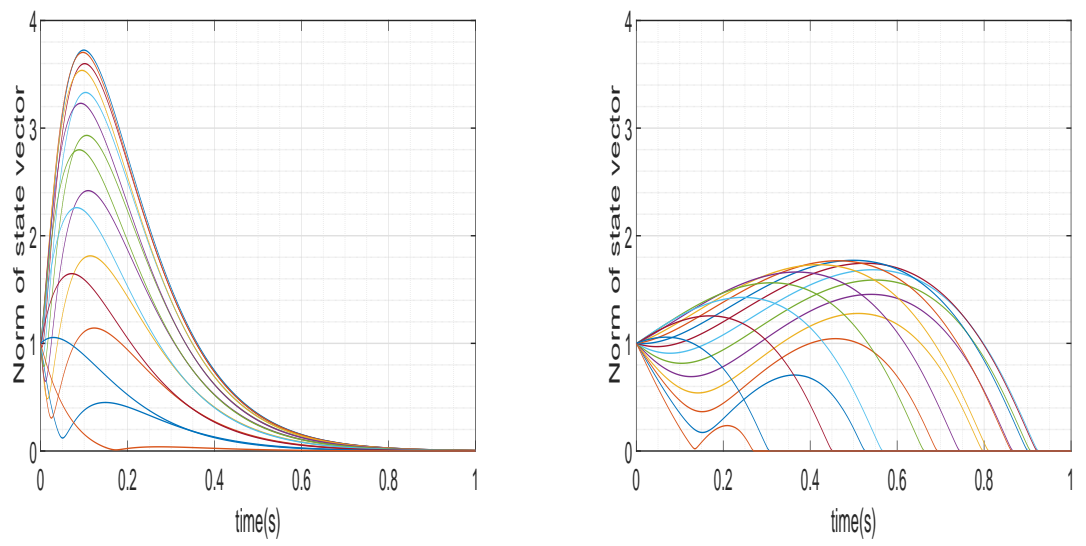

Figure 1: Comparison of "overshoots" for linear (left) and homogeneous (right) controllers

For $x$ belonging to the unit sphere $\|x\|_{\mathbf{d}}=1$, we have $u_{h}(x)=\tilde{k} x$. This means that the homogeneous controller $u_{h}$ is designed by means of a certain homogeneous scaling of a linear stabilizing controller $u(x)=$ $\tilde{k} x$. The aim of this paper is show that an existing linear controller can be "upgraded" to a non-linear one (using the generalized homogeneity) in such a way that the new controller would provide a better control quality (at least, it will never be worst then the linear controller). The main price of this improvement is an additional computational power for the nonlinear control implementation. We develop the design scheme for a linear plant model and confirm our theoretical constructions by real experiments with the quadrotor Q-Drone of Quanser ${ }^{T M}$. To implement the suggested scheme to linear PID controllers we extend the results of [21] to the case of linear geometric dilations and MIMO systems.

\subsection{Notation}

$\mathbb{R}$ denotes the field of real numbers; $\mathbb{C}$ is the field of complex numbers; $\mathbb{R}^{n}$ is the $n$-dimensional Euclidean space with an inner product $\langle\cdot, \cdot\rangle$ and a norm induced by the inner product $\|\cdot\|=\sqrt{\langle\cdot, \cdot\rangle} ; \mathbf{0}$ denotes the zero vector from $\mathbb{R}^{n} ; C(X, Y)$ denotes the space of continuous functions $X \rightarrow Y$, where $X, Y$ are subsets of normed vector spaces; $C^{p}(X, Y)$ is the space of functions continuously differentiable at least up to the order $p ; P \succ 0$ means the matrix $P \in \mathbb{R}^{n \times n}$ is symmetric $P=P^{\top}$ and positive definite; $\lambda_{\min }(P)$ and $\lambda_{\max }(P)$ denotes maximal and minimal eigenvalues of the symmetric matrix $P$; if $A \in \mathbb{R}^{n \times n}$ then the matrix norm of $A$ is defined as follows $\|A\|:=\sup _{x \neq 0} \frac{\|A x\|}{\|x\|}$, where $\|x\|$ is a vector norm in $\mathbb{R}^{n}$.

\section{Generalized Homogeneous Control Systems}

\subsection{Generalized homogeneity}

By definition, homogeneity is a dilation symmetry (see Introduction for more detailed explanations). For example, if a nonlinear function $f: \mathbb{R}^{n} \rightarrow \mathbb{R}$ remains invariant with respect to scaling of its argument

$$
f\left(e^{s} x\right)=e^{\mu s} f(x), \quad s \in \mathbb{R}, \quad x \in \mathbb{R}^{n}
$$

then this function is called (standard) homogeneous, where $\mu \in \mathbb{R}$ is a constant. In general case, instead of the uniform scaling of argument $x \rightarrow e^{s} x$ we can consider a non-uniform one

$$
x \rightarrow \mathbf{d}(s) x
$$


where the operator $\mathbf{d}(s)$ maps $\mathbb{R}^{n}$ onto $\mathbb{R}^{n}$ and $s \in \mathbb{R}$ is a parameter of the dilation. To be a dilation, the family of operators $\mathbf{d}(s)$ must satisfy some conventional restrictions:

- $\mathbf{d}(0) x=x$;

- $\|\mathbf{d}(s) x\| \rightarrow 0$ as $s \rightarrow-\infty$;

- $\|\mathbf{d}(s) x\| \rightarrow+\infty$ as $s \rightarrow+\infty$ for $x \neq 0$.

In [22], [23], [24], the dilation $\mathbf{d}$ is suggested to be generated as a flow of a $C^{1}$ vector field $\nu: \mathbb{R}^{n} \rightarrow \mathbb{R}^{n}$. Namely, let us consider

$$
\dot{\phi}(s)=\nu(\phi(s)), \quad \phi(0)=x, s \in \mathbb{R} .
$$

and assume that the origin of $\dot{z}=-\nu(z)$ is globally uniformly asymptotically stable. The latter means that the origin of (1) is unstable and any solution $\phi_{x}(s)$ with the initial condition $\phi(0)=x \neq \mathbf{0}$ is unique, diverging and can be treated as a dilation of $x$, i.e. $\mathbf{d}(s): \mathbb{R}^{n} \rightarrow \mathbb{R}^{n}$ is defined as

$$
\mathbf{d}(s) x=\phi_{x}(s), \quad x \in \mathbb{R}^{n} .
$$

Indeed, $\mathbf{d}(0) x=x$ and $\|\mathbf{d}(s) x\| \rightarrow+\infty$ as $s \rightarrow+\infty$ for $x \neq 0$ due to instability of (1) in the forward time. Moreover, $\|\mathbf{d}(s) x\| \rightarrow 0$ as $s \rightarrow-\infty$ due to global asymptotic stability of (1) in the backward time.

Such a dilation $\mathbf{d}(s)$ is known as geometric dilation [23], [24]. In this paper we deal only with the socalled linear geometric dilations, which requires the vector fields $\nu$ to be linear, i.e. $\dot{\phi}(s)=G_{\mathbf{d}} \phi(s)$, where $G_{\mathbf{d}} \in \mathbb{R}^{n \times n}$ is an anti-Hurwitz matrix. The matrix $G_{\mathbf{d}}$ is called a generator of the dilation. The dilation $\mathbf{d}$ in this case is given by the matrix exponential

$$
\mathbf{d}(s):=e^{G_{\mathbf{d}} s}=\sum_{i=0}^{\infty} \frac{s^{i} G_{\mathbf{d}}^{i}}{i !} .
$$

Such a type of dilation can be introduced in Banach and Hilbert spaces [3] using the classical theory of (semi)groups of linear bounded operators [25].

Monotonicity of dilation plays an important role for the investigations of homogeneous geometrical structures in $\mathbb{R}^{n}$ as well as for analysis of homogeneous control systems.

Definition 1 The dilation $\mathbf{d}$ is strictly monotone if $\exists \beta>0$ such that

$$
\|\mathbf{d}(s)\| \leq e^{\beta s}, \quad \forall s \leq 0 .
$$

In a finite dimensional space, any dilation is strictly monotone if a norm in $\mathbb{R}^{n}$ is properly selected.

Theorem 1 [26] Let $\mathbf{d}$ be a dilation in the Euclidean space $\mathbb{R}^{n}$ with the inner product

$$
\langle u, v\rangle=u^{\top} P v, \quad u, v \in \mathbb{R}^{n},
$$

where $0 \prec P=P^{\top} \in \mathbb{R}^{n \times n}$ is a positive definite symmetric matrix. The dilation $\mathbf{d}$ is strictly monotone in $\mathbb{R}^{n}$ equipped with the norm $\|z\|=\sqrt{\langle z, z\rangle}$ if and only if the following linear matrix inequality holds

$$
P G_{\mathbf{d}}+G_{\mathbf{d}}^{\top} P \succ 0, \quad P \succ 0
$$

where $G_{\mathbf{d}} \in \mathbb{R}^{n}$ is the generator of the dilation $\mathbf{d}$. Moreover, one has

$$
\begin{aligned}
& e^{\alpha s} \leq\lfloor\mathbf{d}(s)\rfloor \leq\|\mathbf{d}(s)\| \leq e^{\beta s} \quad \text { if } s \leq 0, \\
& e^{\beta s} \leq\lfloor\mathbf{d}(s)\rfloor \leq\|\mathbf{d}(s)\| \leq e^{\alpha s} \quad \text { if } s \geq 0,
\end{aligned}
$$

where

$$
\begin{gathered}
\lfloor\mathbf{d}(s)\rfloor:=\inf _{x \neq \mathbf{0}} \frac{\|\mathbf{d}(s) x\|}{\|x\|}, \\
\alpha=\frac{1}{2} \lambda_{\max }\left(P^{\frac{1}{2}} G_{\mathbf{d}} P^{-\frac{1}{2}}+P^{-\frac{1}{2}} G_{\mathbf{d}}^{\top} P^{\frac{1}{2}}\right)>0
\end{gathered}
$$

and

$$
\beta=\frac{1}{2} \lambda_{\min }\left(P^{\frac{1}{2}} G_{\mathbf{d}} P^{-\frac{1}{2}}+P^{-\frac{1}{2}} G_{\mathbf{d}}^{\top} P^{\frac{1}{2}}\right)>0 .
$$




\subsection{Canonical homogeneous norm}

A dilation allows a sort of a new norm-topology to be introduced using the so-called homogeneous norm.

Definition 2 A continuous function $p: \mathbb{R}^{n} \rightarrow[0,+\infty)$ is said to be a $\mathbf{d}$-homogeneous norm in $\mathbb{R}^{n}$ if

- $p(u) \rightarrow 0$ as $u \rightarrow \mathbf{0}$;

- $p(\mathbf{d}(s) u)=e^{s} p(u)>0$ for $u \in \mathbb{R}^{n} \backslash\{\mathbf{0}\}, s \in \mathbb{R}$;

where $\mathbf{d}$ is a dilation.

The functional $p$ may not satisfy triangle inequality $p(u+v) \leq p(u)+p(v)$, so, formally, it is not even a semi-norm. However, many authors (see e.g. [4], [27], [28]) call the function satisfying the above definition by "homogeneous norm". We follow this tradition. For example, if the dilation is given by $\mathbf{d}(s)=\operatorname{diag}\left\{e^{r_{1} s}, e^{r_{2} s}, \ldots, e^{r_{n} s}\right\}$, a homogeneous norm $p: \mathbb{R}^{n} \rightarrow[0,+\infty)$ can be defined as follows

$$
p(u)=\sum_{i=1}^{n}\left|u_{i}\right|^{\frac{1}{r_{i}}}, \quad u=\left(u_{1}, u_{2}, \ldots, u_{n}\right)^{\top} \in \mathbb{R}^{n} .
$$

For strictly monotone dilations the so-called canonical homogeneous norm [20] can be introduced by means of a homogeneous projection to the unit sphere.

Definition 3 The function $\|\cdot\|_{\mathbf{d}}: \mathbb{R}^{n} \backslash\{\mathbf{0}\} \rightarrow(0,+\infty)$ defined as

$$
\|u\|_{\mathbf{d}}=e^{s_{u}}, \text { where } s_{u} \in \mathbb{R}:\left\|\mathbf{d}\left(-s_{u}\right) u\right\|=1,
$$

is called the canonical homogeneous norm, where $\mathbf{d}$ is a strictly monotone dilation.

Obviously, $\|\mathbf{d}(s) x\|_{\mathbf{d}}=e^{s}\|x\|_{\mathbf{d}}$ and $\|x\|_{\mathbf{d}}=\|-x\|_{\mathbf{d}}$ for any $x \in \mathbb{R}^{n}$ and any $s \in \mathbb{R}$. The homogeneous norm defined by (6) was called canonical since it is induced by a canonical norm $\|\cdot\|$ in $\mathbb{R}^{n}$ and

$$
\|x\|_{\mathbf{d}}=1 \quad \Leftrightarrow \quad\|x\|=1
$$

The monotonicity of the dilation group guarantees that the function $\|\cdot\|_{\mathbf{d}}$ is single-valued and continuous at the origin.

Theorem 2 [20] If $\mathbf{d}$ is a strictly monotone continuous dilation on $\mathbb{R}^{n}$ then

- the function $\|\cdot\|_{\mathbf{d}}: \mathbb{R}^{n} \backslash\{\mathbf{0}\} \rightarrow \mathbb{R}_{+}$given by (6) is single-valued and positive;

- $\|u\|_{\mathbf{d}} \rightarrow 0$ as $u \rightarrow \mathbf{0}$;

- if the norm in $\mathbb{R}^{n}$ is defined as $\|x\|=\sqrt{x^{\top} P x}$ with $P \in \mathbb{R}^{n \times n}$ satisfying (4) then

$$
\frac{\partial\|x\|_{\mathbf{d}}}{\partial x}=\|x\|_{\mathbf{d}} \frac{x^{\top} \mathbf{d}^{\top}\left(-\ln \|x\|_{\mathbf{d}}\right) P \mathbf{d}\left(-\ln \|x\|_{\mathbf{d}}\right)}{x^{\top} \mathbf{d}^{\top}\left(-\ln \|x\|_{\mathbf{d}}\right) P G_{\mathbf{d}} \mathbf{d}\left(-\ln \|x\|_{\mathbf{d}}\right) x}
$$

for any $x \neq \mathbf{0}$.

It is well known (see e.g. [28]) that the norm $\|x\|=\sqrt{x^{\top} P x}$ is a Lyapunov function for any stable linear system $\dot{x}=A x, A \in \mathbb{R}^{n \times n}$. It is expected that the canonical homogeneous norm would be a Lyapunov function for a class of stable homogeneous systems.

\subsection{Homogeneous Systems}

Homogeneity is a dilation symmetry. More formally, it is given by the following definition, which, originally, has been proposed by L. Euler for standard homogeneous functions and by V. Zubov [1] for the weighted homogeneous ones. 
Definition 4 A vector field $f: \mathbb{R}^{n} \rightarrow \mathbb{R}^{n}$ is said to be $\mathbf{d}$-homogeneous of degree $\mu \in \mathbb{R}$ if

$$
f(\mathbf{d}(s) u)=e^{\mu s} \mathbf{d}(s) f(u) \quad \text { for } \quad s \in \mathbb{R}, \quad u \in \mathbb{R}^{n},
$$

where $\mathbf{d}$ is a group of linear operators in $\mathbb{R}^{n}$.

A lot of examples of $\mathbf{d}$-homogeneous non-linear vector fields can be found in the literature [20]. Obviously, any linear vector field $x \rightarrow A x, A \in \mathbb{R}^{n \times n}$ is homogeneous of the zero degree with respect to the standard(uniform) dilation $x \rightarrow e^{s} x$. However, in some cases the linear vector field may be $\mathbf{d}$-homogeneous of positive or negative degree dependently of a concrete dilation $\mathbf{d}$.

Lemma 1 ([26]) Let $\mathbf{d}$ be a continuous group of invertible operators on $\mathbb{R}^{n}$ and $A \in \mathbb{R}^{n \times n}$. The operator $x \rightarrow A x$ with $x \in \mathbb{R}^{n}$ is $\mathbf{d}$-homogeneous of degree $\mu \in \mathbb{R}$ if and only if

$$
A G_{\mathbf{d}}=\left(\mu I+G_{\mathbf{d}}\right) A,
$$

where $G_{\mathbf{d}} \in \mathbb{R}^{n \times n}$ is a generator of $\mathbf{d}$.

Homogeneity allows a local property (e.g. Lipschitz continuity or differentiability) to be extended globally. For example, in [26] it is shown that a d-homogeneous vector field is locally Lipschitz continuous (resp. differentiable) on $\mathbb{R}^{n} \backslash\{\mathbf{0}\}$ if and only if it is Lipschitz continuous (differentiable) on the unit sphere $x^{\top} P x=1$, where $P$ satisfies (4).

Homogeneity of a function (operator or a vector field) is inherited by mathematical object induced by this function such a derivatives or solutions of differential equations. For example, if the right hand side of the following differential equation

$$
\dot{\xi}=f(\xi), \quad t>0, f: \mathbb{R}^{n} \rightarrow \mathbb{R}^{n}
$$

is $\mathbf{d}$-homogeneous of degree $\mu$ then

$$
x_{\mathbf{d}(s) x_{0}}(t)=\mathbf{d}(s) x_{x_{0}}\left(e^{\mu s} t\right), t>0
$$

where $x_{x_{0}}(t), t>0$ denotes a solution of (10) with the initial condition $x(0)=x_{0}$.

Theorem 3 [26] Let $f \in C\left(\mathbb{R}^{n} \backslash\{\mathbf{0}\}, \mathbb{R}^{n}\right)$ be $\mathbf{d}$-homogeneous of degree $\mu \in \mathbb{R}$. The next claims are equivalent.

1) The origin of the system (10) is asymptotically stable.

2) The origin of the system

$$
\dot{z}=\|z\|^{1+\mu}\left(\frac{\left(I_{n}-G_{\mathbf{d}}\right) z^{\top} z P}{z^{\top} P G_{\mathbf{d}} z}+I_{n}\right) f\left(\frac{z}{\|z\|}\right)
$$

is asymptotically stable, where $\|z\|=\sqrt{z^{\top} P z}$ with $P$ satisfying

$$
P G_{\mathbf{d}}+G_{\mathbf{d}}^{\top} P>0, \quad 0<P=P^{\top} \in \mathbb{R}^{n \times n} .
$$

3) For any matrix $P \in \mathbb{R}^{n \times n}$ satisfying (12) there exists a $\mathbf{d}$-homogeneous vector field $\Psi: \mathbb{R}^{n} \rightarrow \mathbb{R}^{n}$ of degree $\mathbf{0}$ such that $\Psi \in \mathbb{C}^{\infty}\left(\mathbb{R}^{n} \backslash\{\mathbf{0}\}, \mathbb{R}^{n}\right)$ is diffemorphism on $\mathbb{R}^{n} \backslash\{\mathbf{0}\}$, homeomorphism on $\mathbb{R}^{n}$, $\Psi(\mathbf{0})=\mathbf{0}$ and

$$
\frac{\partial\left(\Psi^{\top}(\xi) P \Psi(\xi)\right)}{\partial \xi} f(\xi)<0 \quad \text { if } \Psi^{\top}(\xi) P \Psi(\xi)=1 .
$$

Moreover, $\|\Psi\|_{\mathbf{d}} \in \mathbb{H}_{\mathbf{d}}\left(\mathbb{R}^{n}\right) \cap C^{\infty}\left(\mathbb{R}^{n} \backslash\{\mathbf{0}\}\right)$ is Lyapunov function for the system (10), where $\|\cdot\|_{\mathbf{d}}$ is the canonical homogeneous norm induced by $\|\xi\|=\sqrt{\xi^{\top} P \xi}$.

An existence of a smooth homogeneous Lyapunov function for any asymptotically stable homogeneous system is proved in [1], [29]. This result together with the latter theorem implies that any stable dhomogeneous system is topologically equivalent to a quadratically stable standard homogeneous system. Recall that quadratic stability means existence of a quadratic Lyapunov function. All linear stable systems are quadratically stable.

The next proposition characterizes the convergence rates of the homogeneous system. Originally it has been proven in [30] for the weighted dilation. 
Proposition 1 Let $\mathbf{d}$ be a dilation in $\mathbb{R}^{n}$ and $f \in C\left(\mathbb{R} \backslash\{\mathbf{0}\}, \mathbb{R}^{n}\right)$. If the system (10) is $\mathbf{d}$-homogeneous of degree $\mu \in \mathbb{R}$ and its origin is locally uniformly asymptotically stable then

- for $\mu<0$ it is globally uniformly finite-time stable, i.e. there exists a function $T: \mathbb{R}^{n} \rightarrow[0,+\infty)$, which is locally bounded and continuous at $\mathbf{0}$, such that

$$
x_{x_{0}}(t)=0, \quad \forall t \geq T\left(x_{0}\right)
$$

- for $\mu=0$ it is globally uniformly asymptotically stable;

- for $\mu>0$ it is globally uniformly practically fixed-time stable,

$$
\forall r>0, \quad \exists T=T(r)>0 \quad: \quad\left\|x_{x_{0}}(t)\right\|<r, \quad \forall t \geq T, \quad \forall x_{0} \in \mathbb{R}^{n} .
$$

Proof. According to Theorem 3 we can make a change of coordinates $y=\Psi(x)$ such that $\|\cdot\|_{\mathbf{d}}$ is the Lyapunov function for the transformed system

$$
\dot{y}=\tilde{f}(y),
$$

where $\tilde{f}(y)=\left.\frac{\partial \Psi(x)}{\partial x} f(x)\right|_{x=\Psi^{-1}(y)}$ is also $\mathbf{d}$ - homogeneous of degree $\mu$ (due to d-homogeneity of $\Psi$ and $f$ ). Hence, we derive

$$
\frac{d}{d t}\|y\|_{\mathbf{d}}=\|y\|_{\mathbf{d}} \frac{y^{\top} \mathbf{d}^{\top}\left(-\ln \|y\|_{\mathbf{d}}\right) P \mathbf{d}\left(-\ln \|y\|_{\mathbf{d}}\right) \tilde{f}(y)}{y^{\top} \mathbf{d}^{\top}\left(-\ln \|y\|_{\mathbf{d}}\right) P G_{\mathbf{d}} \mathbf{d}\left(-\ln \|y\|_{\mathbf{d}}\right) y}=\|y\|_{\mathbf{d}}^{1+\mu} \frac{y^{\top} \mathbf{d}^{\top}\left(-\ln \|y\|_{\mathbf{d}}\right) P \tilde{f}\left(\mathbf{d}\left(-\ln \|y\|_{\mathbf{d}}\right) y\right)}{y^{\top} \mathbf{d}^{\top}\left(-\ln \|y\|_{\mathbf{d}}\right) P G_{\mathbf{d}} \mathbf{d}\left(-\ln \|y\|_{\mathbf{d}}\right) y}<0,
$$

where the $\mathbf{d}$-homogeneity of $\tilde{f}$ is utilized on the last step. Since $\mathbf{d}\left(-\ln \|y\|_{\mathbf{d}}\right) y$ belong to the unit sphere then taking into account continuity of $\tilde{f}$ we derive

$$
\frac{d}{d t}\|y\|_{\mathbf{d}} \leq-c\|y\|_{\mathbf{d}}^{1+\mu}, \quad c=\inf _{z^{\top} P z=1}-\frac{z^{\top} P \tilde{f}(z)}{z^{\top} P G_{\mathbf{d}} z}>0 .
$$

The obtained differential inequality immediately implies the claimed convergence properties dependently of the homogeneity degree $\mu$.

\subsection{Homogeneous Stabilization of Linear MIMO Systems}

Let us consider the linear control system

$$
\dot{x}=A x+B u(x), \quad t>0,
$$

where $x(t) \in \mathbb{R}^{n}$ is the system state, $u: \mathbb{R}^{n} \rightarrow \mathbb{R}^{m}$ is the feedback control, $A \in \mathbb{R}^{n \times n}$ and $B \in \mathbb{R}^{n \times m}$ are system matrices.

\section{Definition 5 A system}

$$
\dot{x}=f(x, u), t>0, \quad f: \mathbb{R}^{n} \times \mathbb{R}^{m} \rightarrow \mathbb{R}^{n}
$$

is said to be $\mathbf{d}$-homogeneously stabilizable with degree $\mu \in \mathbb{R}$ if there exists a (locally or a globally bounded) feedback law $u: \mathbb{R}^{n} \rightarrow \mathbb{R}^{m}$ such that the closed-loop system is globally asymptotically stable and $\mathbf{d}$-homogeneous of degree $\mu$, where $\mathbf{d}$ is a dilation in $\mathbb{R}^{n}$.

In [31] it is shown that the system (14) can be homogeneously stabilized with a degree $\mu \neq 0$ if and only if the pair $\{A, B\}$ is controllable (or, equivalently, $\operatorname{rank}\left(B, A B, \ldots, A^{n-1} B\right)=n$. We refer the reader to [32] for more details about controllability of linear plants. The following theorem is the corollary of a more general theorem proved [20] for evolution system in Hilbert spaces (see also [31] for more details about the finite dimensional case). 
Theorem 4 If the pair $\{A, B\}$ is controllable and $\mu \in\left[-1, k^{-1}\right]$, where $k \leq n: \operatorname{rank}\left(B, A B, \ldots, A^{k-1} B\right)=n$, then a homogeneously stabilizing control for (14) can always be selected in the form

$$
u(x)=K_{0} x+\|x\|_{\mathbf{d}}^{1+\mu} Y X^{-1} \mathbf{d}\left(-\ln \|x\|_{\mathbf{d}}\right) x
$$

with any $K_{0} \in \mathbb{R}^{n \times m}$ such that $A_{0}=A+B K_{0}$ is nilpotent, any dilation $\mathbf{d}$ generated by $G_{\mathbf{d}} \in \mathbb{R}^{n \times n}$ satisfying

$$
A_{0} G_{\mathbf{d}}=\left(G_{\mathbf{d}}+\mu I\right) A_{0}, \quad G_{\mathbf{d}} B=B
$$

and any $X \in \mathbb{R}^{n \times n}, Y \in \mathbb{R}^{m \times n}$ solving the following algebraic system

$$
\left\{\begin{array}{l}
X A_{0}^{\top}+A_{0} X+Y^{\top} B^{\top}+B Y+X G_{\mathbf{d}}^{\top}+G_{\mathbf{d}} X=\mathbf{0} \\
X G_{\mathbf{d}}^{\top}+G_{\mathbf{d}} X \succ 0, \quad X \succ 0
\end{array}\right.
$$

where the canonical homogeneous norm $\|\cdot\|_{\mathbf{d}}$ is induced by the norm $\|x\|=\sqrt{x^{\top} X^{-1} x}$.

The canonical homogeneous norm is a Lyapunov function of the closed-loop system (14), (15) and

$$
\frac{d}{d t}\|x(t)\|_{\mathbf{d}}=-\|x(t)\|_{\mathbf{d}}^{1+\mu} \quad \text { for } \quad t>0: x(t) \neq \mathbf{0} .
$$

If $A_{0}$ is nilpotent, then (16) has a solution with respect to $G_{\mathbf{d}}$ [31], such that $G_{\mathbf{d}}$ is anti-Hurwitz matrix. The feasibility of (17) is proven in [18] and refined in [31]. The proof of the latter theorem follows from the following computations

$$
\begin{gathered}
\frac{d}{d t}\|x\|_{\mathbf{d}}= \\
\frac{\partial\|x\|_{\mathbf{d}}}{\partial x} \dot{x}=\|x\|_{\mathbf{d}} \frac{x^{\top} \mathbf{d}^{\top}\left(-\ln \|x\|_{\mathbf{d}}\right) X^{-1} \mathbf{d}\left(-\ln \|x\|_{\mathbf{d}}\right)\left(A_{0} x+\|x\|_{\mathbf{d}}^{1+\mu} B Y X^{-1} \mathbf{d}\left(-\ln \|x\|_{\mathbf{d}}\right) x\right)}{x^{\top} \mathbf{d}^{\top}\left(-\ln \|x\|_{\mathbf{d}}\right) X^{-1} G_{\mathbf{d}} \mathbf{d}\left(-\ln \|x\|_{\mathbf{d}}\right) x},
\end{gathered}
$$

where the formula (7) is utilized on the last step. Indeed, from (16) we derive d-homogeneity of $A_{0}$ (namely, $A_{0} \mathbf{d}(s)=e^{\mu s} \mathbf{d}(s) A_{0}$ for any $s \in \mathbb{R}$ ) and $B$ (namely, $\mathbf{d}(s) B=e^{s} B$ for all $s \in \mathbb{R}$ ). Hence, using (16) we immediately derive

$$
\frac{d}{d t}\|x\|_{\mathbf{d}}=\|x\|_{\mathbf{d}}^{1+\mu} \frac{\left.x^{\top} \mathbf{d}^{\top}\left(-\ln \|x\|_{\mathbf{d}}\right) X^{-1}\left(A_{0}+B Y X^{-1}\right) \mathbf{d}\left(-\ln \|x\|_{\mathbf{d}}\right) x\right)}{x^{\top} \mathbf{d}^{\top}\left(-\ln \|x\|_{\mathbf{d}}\right) X^{-1} G_{\mathbf{d}} \mathbf{d}\left(-\ln \|x\|_{\mathbf{d}}\right) x}=-\|x\|_{\mathbf{d}}^{1+\mu}
$$

for $x \neq \mathbf{0}$.

Remark 1 If the matrix A is nilpotent (like in the example about the "peaking effect" given in the introduction) then $K_{0}=\mathbf{0}$. In this case, for $\mu=-1$ any solution $x\left(t, x_{0}\right)$ of the closed loop system (14), (15) with the initial condition $x(0)=x_{0}$ satisfies

$$
x\left(t, x_{0}\right)=0, \quad \forall t \geq\left\|x_{0}\right\|_{\mathbf{d}}
$$

and

$$
\|u(x)\|=\left\|Y X^{-1} \mathbf{d}\left(-\ln \|x\|_{\mathbf{d}}\right) x\right\| \leq\left\|Y X^{-1}\right\| \cdot\left\|\mathbf{d}\left(-\ln \|x\|_{\mathbf{d}}\right) x\right\|=\left\|Y X^{-1}\right\|<+\infty .
$$

The latter means that the presented homogeneous controller solves the stabilization problem without an unbounded "peaking effect" (see the introduction for more details). To guarantee that the settling time of the closed-loop system is bounded by a number $T>0$, for all $x_{0}^{T} x_{0} \leq 1$, we just need to add the following linear matrix inequality

$$
\mathbf{d}(\ln T) X \mathbf{d}^{\top}(\ln T) \preceq I_{n}
$$

to the system (17). The extended system of LMIs remains feasible. Indeed, if the pair $X_{0}, Y_{0}$ is a solution of (17) then for any $q>0$ the pair $X=q X_{0}, Y=q Y_{0}$ is a solution as well. Hence, the matrix inequality (18) is fulfilled for a sufficiently small $q>0$.

The following corollary study the case of the perturbed linear system. The perturbations can be modeled by a set-valued or a discontinuous function (e.g. dry friction) provided that Filippov solution exists [33]. 
Corollary 1 Let conditions of Theorem 4 hold and $F: \mathbb{R} \times \mathbb{R}^{n} \rightrightarrows \mathbb{R}^{n}$ satisfy the following inequality

$$
\frac{\sup _{y \in F(t, x)}\left\|\mathbf{d}\left(-\ln \|x\|_{\mathbf{d}}\right) y\right\|}{x^{\top} \mathbf{d}^{\top}\left(-\ln \|x\|_{\mathbf{d}}\right) P G_{\mathbf{d}} \mathbf{d}\left(-\ln \|x\|_{\mathbf{d}}\right) x} \leq \kappa\|x\|_{\mathbf{d}}^{\mu}, \quad \forall x \in \mathbb{R}^{n} \backslash\{\mathbf{0}\}, \forall t \geq 0
$$

for some $\kappa>0$, where the canonical homogeneous norm $\|\cdot\|_{\mathbf{d}}$ is induced by $\|x\|=\sqrt{x^{\top} P x}, P=X^{-1}$. If, additionally,

$$
(A+B K)^{\top} P+P(A+B K)+(\rho+\kappa)\left(G_{\mathbf{d}}^{\top} P+P G_{\mathbf{d}}\right) \preceq 0, \quad \rho>0,
$$

and $F$ is compact-valued, convex-valued and upper-semi continuous then the control (15) stabilize the system

$$
\dot{x} \in A x+B u(x)+F(t, x), t>0
$$

and

$$
\frac{d}{d t}\|x(t)\|_{\mathbf{d}} \leq-\rho\|x(t)\|_{\mathbf{d}}^{1+\mu}
$$

Proof. The existence of solutions of closed-loop system follows from Filippov theory [33]. Stability immediately follows the following computations

$$
\begin{gathered}
\frac{d}{d t}\|x\|_{\mathbf{d}}=\|x\|_{\mathbf{d}} \frac{\left.x^{\top} \mathbf{d}^{\top}\left(-\ln \|x\|_{\mathbf{d}}\right) X^{-1} \mathbf{d}\left(-\ln \|x\|_{\mathbf{d}}\right) \dot{x}\right)}{x^{\top} \mathbf{d}^{\top}\left(-\ln \|x\|_{\mathbf{d}}\right) X^{-1} G_{\mathbf{d}} \mathbf{d}\left(-\ln \|x\|_{\mathbf{d}}\right) x} \leq \\
\|x\|_{\mathbf{d}}^{1+\mu} \frac{\left.x^{\top} \mathbf{d}^{\top}\left(-\ln \|x\|_{\mathbf{d}}\right) X^{-1}\left(A_{0}+B Y X^{-1}\right) \mathbf{d}\left(-\ln \|x\|_{\mathbf{d}}\right) x\right)}{x^{\top} \mathbf{d}^{\top}\left(-\ln \|x\|_{\mathbf{d}}\right) X^{-1} G_{\mathbf{d}} \mathbf{d}\left(-\ln \|x\|_{\mathbf{d}}\right) x}+ \\
\|x\|_{\mathbf{d}} \sup _{y \in F(t, x)} \frac{x^{\top} \mathbf{d}^{\top}\left(-\ln \|x\|_{\mathbf{d}}\right) X^{-1} \mathbf{d}\left(-\ln \|x\|_{\mathbf{d}}\right) y}{x^{\top} \mathbf{d}^{\top}\left(-\ln \|x\|_{\mathbf{d}}\right) X^{-1} G_{\mathbf{d}} \mathbf{d}\left(-\ln \|x\|_{\mathbf{d}}\right) x} \leq \\
-(\rho+\kappa)\|x\|_{\mathbf{d}}^{\mu+1}+\kappa\|x\|_{\mathbf{d}}^{1+\mu} \leq-\rho\|x\|_{\mathbf{d}}^{1+\mu}
\end{gathered}
$$

Notice that the obtained differential inequality obtained for the canonical homogeneous norm specifies the convergence rate of the closed-loop system.

\subsection{Homogeneous Proportional Integral Controller}

The linear control theory uses an integral terms in order to improve robustness properties of a proportional feedback law. This idea is also useful for nonlinear controllers [34]. A similar integrator can be added to implicit homogeneous feedback [21].

Theorem 5 Let $K_{0} \in \mathbb{R}^{m \times n}$ be such that $A+B K_{0}$ is nilpotent, rank $(B)=m$ and an anti-Hurwitz matrix $G_{\mathbf{d}} \in \mathbb{R}^{n \times n}$ satisfy (16) with $\mu \in[-0.5,1 / k]$, where the number $k$ is given in Theorem 4 .

Let $X \in \mathbb{R}^{n \times n}$ and $Y \in \mathbb{R}^{m \times n}$ satisfy (17) then for any positive definite matrix $Q \in \mathbb{R}^{m \times m}$ and any constant vector $p \in \mathbb{R}^{m}$ the control law

$$
\begin{gathered}
u(x)=K_{0} x+u_{h o m}(x)+\int_{0}^{t} u_{i n t}(x(s)) d s, \\
u_{h o m}(x)=\|x\|_{\mathbf{d}}^{1+\mu} Y X^{-1} \mathbf{d}\left(-\ln \|x\|_{\mathbf{d}}\right) x, \\
u_{\text {int }}(x)=-\|x\|_{\mathbf{d}}^{1+2 \mu} \frac{Q B^{\top} P \mathbf{d}\left(-\ln \|x\|_{\mathbf{d}}\right) x}{x^{\top} \mathbf{d}^{\top}\left(-\ln \|x\|_{\mathbf{d}}\right) P G_{\mathbf{d}} \mathbf{d}\left(-\ln \|x\|_{\mathbf{d}}\right) x}
\end{gathered}
$$

stabilizes the origin of the system

$$
\dot{x}=A x+B(u+p),
$$

in a finite time if $\mu<0$, exponentially if $\mu=0$, and practically in a fixed-time if $\mu>0$, where, as before, the canonical homogeneous norm $\|\cdot\|_{\mathbf{d}}$ is induced by the norm $\|x\|=\sqrt{x^{\top} X^{-1} x}$. 
Proof. Let us introduce the following virtual variable

$$
x_{n+1}=p+\int_{0}^{t} u_{i n t}(x(s)) d s .
$$

In this case the closed-loop system becomes

$$
\dot{x}=A x+B\left(K_{0} x+u_{\text {hom }}(x)+x_{n+1}\right), \quad \dot{x}_{n+1}=u_{\text {int }}(x) .
$$

Since $\left\|\mathbf{d}\left(-\ln \|x\|_{\mathbf{d}}\right) x\right\|=1$ then $u_{\text {int }}$ is globally bounded and discontinuous at $x=\mathbf{0}$ if $\mu=-0.5$. In all other cases, the considered system has the continuous right-hand side.

Let us show that the latter system is globally asymptotically stable. For this purpose let us consider the following Lyapunov function candidate

$$
V=\frac{1}{2+2 \mu}\|x\|_{\mathbf{d}}^{2+2 \mu}+\frac{1}{2} x_{n+1}^{\top} Q^{-1} x_{n+1} .
$$

Calculating the time derivative of $V$ along the trajectories of the closed-loop system we derive

$$
\begin{gathered}
\dot{V}=\|x\|_{\mathbf{d}}^{1+2 \mu} \frac{\partial\|x\|_{\mathbf{d}}}{\partial x} \dot{x}+x_{n+1}^{\top} Q^{-1} \dot{x}_{n+1}= \\
-\|x\|_{\mathbf{d}}^{2+3 \mu}+\|x\|_{\mathbf{d}}^{1+2 \mu} \frac{\partial\|x\|_{\mathbf{d}}}{\partial x} B x_{n+1}-\frac{\|x\|^{1+2 \mu} x_{n+1}^{\top} B^{\top} P \mathbf{d}\left(-\ln \|x\|_{\mathbf{d}}\right) x}{x^{\top} \mathbf{d}^{\top}\left(-\ln \|x\|_{\mathbf{d}}\right) P G_{\mathbf{d}} \mathbf{d}\left(-\ln \|x\|_{\mathbf{d}}\right) x}=-\|x\|_{\mathbf{d}}^{2+3 \mu},
\end{gathered}
$$

where the formula (7) and the identity $e^{s G_{\mathbf{d}}} B=e^{s} B$ is utilized on the last step.

Since $x=0, x_{n+1}=0$ is the unique equilibrium of the system $(23)$ and the hyperplane $\left\{\left(x, x_{n+1}\right) \in\right.$ $\left.\mathbb{R}^{n+m}: x=\mathbf{0}\right\}$ does not contain non-zero trajectories of this system, then its origin is globally asymptotically stable (see, e.g. LaSalle principle [35] and its version for discontinuous ODEs [36]).

Finally, since the system (23) is $\mathbf{d}$-homogeneous of the degree $\mu$ with respect to the dilation

$$
\tilde{\mathbf{d}}(s)=\left(\begin{array}{cc}
e^{s G_{\mathbf{d}}} & \mathbf{0} \\
\mathbf{0} & e^{s(1+\mu)} I_{m}
\end{array}\right)
$$

then using Proposition 1 we complete the proof.

In the case of the weighted homogeneous SISO system (14) the presented theorem with the degree $\mu=-0.5$ recovers the result of [21].

Remark 2 Since the functional

$$
x \rightarrow x^{\top} \mathbf{d}^{\top}\left(-\ln \|x\|_{\mathbf{d}}\right) P G_{\mathbf{d}} \mathbf{d}\left(-\ln \|x\|_{\mathbf{d}}\right) x
$$

is $\mathbf{d}$-homogeneous of the degree 0 and uniformly bounded from above and from below then its replacement in $u_{\text {int }}$ with a constant does not destroy the homogeneity properties of the system. Therefore, for practical reasons the simplified integral term

$$
u_{\text {int }}(x)=-\|x\|_{\mathbf{d}}^{1+2 \mu} Q B^{\top} P \mathbf{d}\left(-\ln \|x\|_{\mathbf{d}}\right) x
$$

can be utilized provided that the stability of the closed-loop system is preserved. This replacement could add some additional restrictions to parameters $Q, X$ and $\mu$. Some sufficient conditions of the quadratic-like stability of nonlinear generalized homogeneous systems presented in [26] can be utilized for the corresponding analysis. The stability of the obtained nonlinear system can also be studied using, for example, robustness properties of the homogeneous proportional integral controller (see [21] for more details about its robustness in the case of the weighted homogeneity). The derivation of an LMI-based conditions allowing the simplified form of the integral term is an interesting theoretical problem for the future research.

\section{Homogenization of Linear Controllers}

The PID (Proportional-Integral-Derivative) controller is the most common linear feedback law for real physical control systems. The previous sections shown that homogeneous systems may have a better robustness properties and faster convergence rate. In this section the question to be studied is : Is it possible to upgrade an existing linear (in particular PID) controller in order to make a closed-loop locally or globally d-homogeneous and improve convergence properties of the system? A scheme of the upgrade must prevent a possible degradation of the control quality and only allow its improvement. 


\subsection{Design of a homogeneous controller from an existing linear feedback}

Consider again the linear system (14) and assume that some linear control law

$$
u_{l i n}(x)=K_{l i n} x, \quad K_{l i n} \in \mathbb{R}^{m \times n}, \quad x \in \mathbb{R}^{n}
$$

is already designed.

Corollary 2 Let the pair $\{A, B\}$ be controllable, $K_{0} \in \mathbb{R}^{m \times n}$ be such that the matrix $A_{0}=A+B K_{0}$ is nilpotent and $K_{\text {lin }} \in \mathbb{R}^{m \times n}$ be such that the matrix $A+B K_{\text {lin }}$ is Hurwitz.

Let $G_{\mathbf{d}} \in \mathbb{R}^{n \times n}$ be a generator of the dilation $\mathbf{d}$ such that $(16)$ holds for $\mu=-1$. If a matrix $P=P^{\top} \in$ $\mathbb{R}^{n \times n}$ satisfies the system of linear matrix inequalities

$$
\begin{aligned}
& \left(A+B K_{l i n}\right)^{\top} P+P\left(A+B K_{l i n}\right) \prec 0 \\
& G_{\mathbf{d}}^{\top} P+P G_{\mathbf{d}} \succ 0, \quad P \succ 0
\end{aligned}
$$

then the control $u$ given by (15) with $\mu=-1$ and $K=K_{\text {lin }}-K_{0} \mathbf{d}$-homogeneously stabilizes the origin of the system (14) in a finite-time, where $\|\cdot\|_{\mathbf{d}}$ is the canonical homogeneous norm induced by the norm $\|x\|=\sqrt{x^{\top} P x}$. Moreover, $u_{\text {lin }}(x)=u(x)$ for $x \in S=\left\{x \in \mathbb{R}^{n}:\|x\|=1\right\}$.

The proof immediately follows from the identity

$$
\left(A+B K_{\text {lin }}\right)^{\top} P+P\left(A+B K_{\text {lin }}\right)=\left(A_{0}+B K\right)^{\top} P+P\left(A_{0}+B K\right)
$$

and Theorem 4. Finally, for $\|x\|=1$ we have $\|x\|_{\mathbf{d}}=1, \mathbf{d}\left(-\ln \|x\|_{\mathbf{d}}\right)=\mathbf{d}(0)=I_{n}$, i.e. $u_{\text {lin }}(x)=u(x)$ if $\|x\|=1$.

The proven corollary shows that if a linear controllable plant is exponentially stabilized by means of a linear feedback then it can also be homogeneously stabilized by means of the control (15) using the gains of the original linear controller. These two controllers coincide on the unit sphere $x^{\top} P x=1$. Notice that the corresponding sphere can be always adjusted (if needed) by means of a variation of $P$ satisfying (24).

Let the saturation function $\operatorname{sat}_{a, b}: \mathbb{R}_{+} \rightarrow \mathbb{R}_{+}$be given by

$$
\operatorname{sat}_{a, b}(\rho)=\left\{\begin{array}{ccc}
b & \text { if } & \rho \geq b, \\
\rho & \text { if } & a<\rho<b, \\
a & \text { if } & \rho<a,
\end{array} \quad \rho \in \mathbb{R}_{+} .\right.
$$

Let us consider the control law

$$
u_{a, b}(x)=K_{0} x+K \mathbf{d}\left(-\ln \operatorname{sat}_{a, b}\left(\|x\|_{\mathbf{d}}\right)\right) x,
$$

where $\mathbf{d},\|x\|_{\mathbf{d}}, K_{0}$ and $K=K_{\text {lin }}-K_{0}$ are defined in Corollary 2. The controller (26) also stabilize the origin of the system (14) in the view of results given below.

From (25) we conclude that

$$
u_{1,1}(x)=K_{l i n} x, \quad \forall x \in \mathbb{R}^{n}
$$

and

$$
u_{0,+\infty}(x)=K_{0} x+K \mathbf{d}\left(-\ln \|x\|_{\mathbf{d}}\right) x, \quad \forall x \in \mathbb{R}^{n} .
$$

In other words, the pair $a \in(0,1]$ and $b \in[1,+\infty)$ parametrize a family of non-linear controllers which has the linear and homogeneously stabilizing feedbacks as the limit cases.

Notice that for $b=1$ the controller (26) coincide with the linear controller outside the unit ball $x^{\top} P x>1$ and the gains of the linear controller are scaled by means of dilation $\mathbf{d}$ only close to the origin, i.e. for $x^{\top} P x<1$.

The following scheme for an "upgrade" of linear control to non-linear (locally homogeneous) one can be suggested and the algorithm for an "upgrade" of a linear stabilizing controller to a non-linear homogeneous one:

- Find a matrix $K_{0} \in \mathbb{R}^{m \times n}$ such that $A+B K_{0}$ is nilpotent. 
- Find a symmetric matrix $P=P^{\top}$ satisfying the inequalities (24), which is required to define the canonical homogeneous norm $\|\cdot\|_{\mathbf{d}}$.

- Select $a=b=1$ (i.e. we start with a linear controller).

- Increase $b>1$ and decrease $\alpha<1$ while this improves a quality of the control system.

Theoretically, an improvement of control quality (faster transitions or better robustness) is proved by Corollary 1 even for the case $\alpha=0$ and $\beta=+\infty$. However, the proofs of the corollaries are model-based, but any model of a system is just an approximation of the reality. In practice, a difference between a dynamic model and a real motion of the system may not allow to realize all theoretical properties of the closed-loop system or, even more, it may imply a serious degradation of some performance indexes, which characterizes the control quality. That is why, the tuning of parameters $a$ and $b$ suggested above is required to guarantee that the non-linear control always have the quality which is never worse than the original linear one. It would allow a control engineer to prevent any possible degradation of the control quality during the non-linear "upgrade" of a linear control system. Below the real experiment tested on quadrotor will be presented.

Notice that if the gains of the linear controller are already optimally adjusted, then improvements provided by homogeneous controller could not be huge and the parameters $a$ and $b$ could, possibly, be close 1 in this case. If small variations of the parameters $a$ and $b$ from 1 imply degradations of the control quality then the proposed "upgrade" is impossible.

\subsection{On digital realization of implicit homogeneous feedback}

In order to implement an implicit homogeneous control in practice, an algorithm for computation of the canonical homogeneous norm is required. This norm can be computed explicitly for $n \leq 2$ or approximated by an explicit homogeneous norm for $n \geq 3$ (see [26]. However, even for the second order case the representation of the canonical homogeneous norm is rather cumbersome, so a digital realization of the homogeneous control law requires much more computational power than the linear algorithm. Therefore, an algorithm of a digital realization of the implicit homogeneous control is required for its successful practical application. Some additional properties of the implicit homogeneous controller are established below for this purpose.

Theorem 6 If all conditions of Corollary 1 hold for $G_{\mathbf{d}_{1}}=G_{\mathbf{d}}-I_{n}$ (as in Theorem 4) then for any fixed $r>0$ the closed $\mathbf{d}$-homogeneous ball $\bar{B}_{\mathbf{d}}(r)$ is a strictly positively invariant compact set ${ }^{1}$ of the closed-loop system (21) with the linear control

$$
u_{r}(x)=r^{1+\mu} K \mathbf{d}(-\ln r) x .
$$

Proof. Let us denote

$$
K_{r}=r^{1+\mu} K \mathbf{d}(-\ln r), \quad P_{r}=\mathbf{d}^{\top}(-\ln r) P \mathbf{d}(-\ln r), \quad \rho_{r}=r^{\mu} \rho, \quad \kappa_{r}=r^{\mu} \kappa .
$$

In this case multiplying (20) on $\mathbf{d}^{\top}(-\ln r)$ from the left and on $\mathbf{d}(-\ln r)$ from the right we derive

$$
\begin{gathered}
\mathbf{d}^{\top}(-\ln r)(A+B K)^{\top} \mathbf{d}^{\top}(\ln r) P_{r}+P_{r} \mathbf{d}(\ln r)(A+B K) \mathbf{d}(-\ln r)+ \\
(\rho+\kappa)\left(\mathbf{d}^{\top}(-\ln r) G_{\mathbf{d}}^{\top} \mathbf{d}^{\top}(\ln r) P_{r}+P_{r} \mathbf{d}(\ln r) G_{\mathbf{d}} \mathbf{d}(-\ln r)\right) \preceq 0 .
\end{gathered}
$$

Taking into account $G_{\mathbf{d}} \mathbf{d}(-\ln r)=\mathbf{d}(-\ln r) G_{\mathbf{d}}, A \mathbf{d}(-\ln r)=r^{-\mu} \mathbf{d}(-\ln r) A$ and $\mathbf{d}(\ln r) B=r B$ we derive

$$
r^{-\mu}\left[\left(A+B K_{r}\right)^{\top} P_{r}+P_{r}\left(A+B K_{r}\right)\right]+(\rho+\kappa)\left(G_{\mathbf{d}}^{\top} P_{r}+P_{r} G_{\mathbf{d}}\right) \leq 0
$$

or, equivalently,

$$
\left(A+B K_{r}\right)^{\top} P_{r}+P_{r}\left(A+B K_{r}\right)+\left(\rho_{r}+\kappa_{r}\right)\left(G_{\mathbf{d}}^{\top} P_{r}+P_{r} G_{\mathbf{d}}\right) \preceq 0 .
$$

Hence, the time derivative of the Lyapunov function candidate $V(x)=x^{T} P_{r} x, x \in \mathbb{R}^{n}$ along a trajectory of the closed-loop linear system we have

$$
\dot{V}(x(t))=x^{\top}(t)\left[P_{r}\left(A+B K_{r}\right)+\left(A+B K_{r}\right)^{\top} P_{r}+\rho_{r}\left(G_{\mathbf{d}}^{\top} P_{r}+P_{r} G_{\mathbf{d}}\right)\right] x(t)+
$$

\footnotetext{
${ }^{1} \mathrm{~A}$ set $\Omega$ is said to be a strictly positively invariant for a dynamical system if $x\left(t_{0}\right) \in \Omega \Rightarrow x(t) \in$ int $\Omega, t \geq t_{0}$, where $x$ denotes a solution $x$ of this system.
} 


$$
2 f^{\top}(t) P_{r} x(t)-\rho_{r} x^{\top}(t)\left(P_{r} G_{\mathbf{d}}+G_{\mathbf{d}}^{\top} P_{r}\right) x(t)
$$

where $f(t) \stackrel{\text { a.e. }}{\in} F(t, x(t))$. For $\|x(t)\|_{\mathbf{d}}=r$ from (19) we derive

$$
2 f^{\top}(t) P_{r} x(t) \leq 2 \sqrt{f^{\top}(t) P_{r} f(t)} \leq \kappa_{r} x^{\top}(t)\left(P_{r} G_{\mathbf{d}}+G_{\mathbf{d}}^{\top} P_{r}\right) x(t) .
$$

Hence, we conclude that $\dot{V}(x) \leq-\rho_{r} x^{\top}\left(P_{r} G_{\mathbf{d}}+G_{\mathbf{d}}^{\top} P_{r}\right) x<0$ if $\|x(t)\|_{\mathbf{d}}=r$ (or, equivalently, if $\left.V(x)=1\right)$. The latter immediately implies that $B_{\mathbf{d}}(r)$ is strictly positively invariant set of the closed-loop linear system.

Now we assume that the value $\|x(t)\|_{\mathbf{d}}$ can be changed only in some sampled instances of time and let us show that the corresponding linear switched feedback robustly stabilizes the perturbed linear system.

\section{Corollary 3 If}

1) the conditions of Corollary 1 hold;

2) $\left\{t_{i}\right\}_{i=0}^{+\infty}$ is an arbitrary sequence of time instances such that

$$
0=t_{0}<t_{1}<t_{2}<\ldots \quad \text { and } \quad \lim _{i \rightarrow+\infty} t_{i}=+\infty
$$

3) the switched control $u$ has the form

$$
u(x(t))=\left\|x\left(t_{i}\right)\right\|_{\mathbf{d}}^{1+\mu} K \mathbf{d}\left(-\ln \left\|x\left(t_{i}\right)\right\|_{\mathbf{d}}\right) x(t), \quad t \in\left[t_{i}, t_{i+1}\right)
$$

then the closed-loop system (21), (28) is globally asymptotically stable.

Proof.

I. Let us show that the sequence $\left\{\left\|x\left(t_{i}\right)\right\|_{\mathbf{d}}\right\}_{i=1}^{+\infty}$ is monotone decreasing along any solution of the closedloop system. Notice that the function $t \rightarrow\|x(t)\|_{\mathbf{d}}$ is continuous since the solution $x$ of the closed-loop system is a continuous function of time.

Let us define the quadratic positive definite function $V_{i}: \mathbb{R}^{n} \rightarrow R_{+}$given by $V_{i}(x):=x^{T} P_{i} x$, where $P_{i}:=\mathbf{d}^{\top}\left(-\ln \left\|x\left(t_{i}\right)\right\|_{\mathbf{d}}\right) P \mathbf{d}\left(-\ln \left\|x\left(t_{i}\right)\right\|_{\mathbf{d}}\right)>0$.

On the time interval $\left[t_{i}, t_{i+1}\right)$ we have $u(x)=K_{i} x$, where

$$
K_{i}:=\left\|x\left(t_{i}\right)\right\|_{\mathbf{d}}^{1+\mu} K \mathbf{d}\left(-\ln \left\|x\left(t_{i}\right)\right\|_{\mathbf{d}}\right) .
$$

Repeating the proof of Theorem 6 we derive

$$
\dot{V}_{i}(x(t)) \leq-\rho\left\|x\left(t_{i}\right)\right\|_{\mathbf{d}}^{\mu} x(t)^{T}\left(P_{i} G_{\mathbf{d}}+G_{\mathbf{d}}^{\top} P_{i}\right) x(t)<0
$$

for $t \in\left[t_{i}, t_{i+1}\right)$, i.e. the function $t \rightarrow V_{i}(x(t))$ is strictly decreasing on $\left[t_{i}, t_{i+1}\right)$.

On the one hand, for any fixed $x \neq 0$ the scalar-valued function $r \rightarrow q(r)$ defined as

$$
q(r)=x^{\top} \mathbf{d}^{\top}(-\ln r) P \mathbf{d}(-\ln r) x, \quad r>0
$$

is also strictly decreasing due to $G_{\mathbf{d}}^{\top} P+P G_{\mathbf{d}}>0$. On the other hand, from the definition of the canonical homogeneous norm $\|\cdot\|_{\mathbf{d}}$ we derive $V_{i}\left(x\left(t_{i}\right)\right)=1$ and $\forall t \in\left(t_{i}, t_{i+1}\right]$ we have

$$
\begin{gathered}
V_{i}(x(t))-1=x^{\top}(t) \mathbf{d}^{\top}\left(-\ln \left\|x\left(t_{i}\right)\right\|_{\mathbf{d}}\right) P\left(-\ln \left\|x\left(t_{i}\right)\right\|_{\mathbf{d}}\right) x(t)-1<0= \\
x^{\top}(t) \mathbf{d}^{\top}\left(-\ln \|x(t)\|_{\mathbf{d}}\right) P\left(-\ln \|x(t)\|_{\mathbf{d}}\right) x(t)-1 .
\end{gathered}
$$

The latter implies $\|x(t)\|_{\mathbf{d}}<\left\|x\left(t_{i}\right)\right\|_{\mathbf{d}}$ for all $t \in\left(t_{i}, t_{i+1}\right]$, i.e. the sequence $\left\{\left\|x\left(t_{i}\right)\right\|_{\mathbf{d}}\right\}_{i=1}^{+\infty}$ is monotone decreasing and $x(t) \in B_{\mathbf{d}}\left(\left\|x\left(t_{i}\right)\right\|_{\mathbf{d}}\right)$ for all $t \geq t_{i}$. Moreover, $V(x(t)) \leq V(x(0))$ for all $t \geq 0$, i.e. the origin of the closed-loop system is Lyapunov stable.

II. Since the canonical homogeneous norm $\|\cdot\|_{\mathbf{d}}$ is positive definite then the monotone decreasing sequence $\left\{\left\|x\left(t_{i}\right)\right\|_{\mathbf{d}}\right\}_{i=1}^{\infty}$ converges to some limit. Let us show now that this limit is zero. Suppose the contrary, i.e. $\lim _{i \rightarrow \infty}\left\|x\left(t_{i}\right)\right\|_{\mathbf{d}}=V_{*}>0$ or equivalently $\forall \varepsilon>0 \quad \exists N=N(\varepsilon): V_{*} \leq\left\|x\left(t_{i}\right)\right\|_{\mathbf{d}}<V_{*}+\varepsilon, \forall i \geq N$.

The control function $u(V, s)$ is continuous $\forall s \in \mathbb{R}^{n} \backslash\{0\}$ and $\forall V \in \mathbb{R}_{+}$. The latter means

$$
\|\| x\left(t_{i}\right)\left\|_{\mathbf{d}}^{\mu+1} K \mathbf{d}\left(-\ln \left\|x\left(t_{i}\right)\right\|_{\mathbf{d}}\right) x-V_{*}^{\mu+1} K \mathbf{d}\left(-\ln V^{*}\right) x\right\| \leq \sigma(\varepsilon)\|s\|, \quad \forall i \geq N,
$$


where $\sigma(\cdot) \in \mathcal{K}$. The definition of $\|$ can be found in [37]. This means that for $t>t_{N}$ the closed-loop system can be presented in the form

$$
\dot{x}(t)=\left(A+B\left(K_{*}+\Delta(t, \varepsilon)\right) x+f(t),\right.
$$

where $K_{*}=V_{*}^{1+\mu} K \mathbf{d}\left(-\ln V_{*}\right), f(t) \in F(t, x(t))$ and $\Delta(t, \varepsilon) \in \mathbb{R}^{m \times n}:\|\Delta\| \leq \sigma(\varepsilon)$.

Let us consider the quadratic positive definite Lyapunov function candidate $V_{*}(x)=x^{T} P_{*} x$, where $P_{*}=\mathbf{d}^{\top}\left(-\ln V^{*}\right) P \mathbf{d}\left(-\ln V^{*}\right)$. For $t>t_{N}$ we have

$$
\begin{gathered}
\dot{V}_{*}(x(t)) \leq-(\rho+\kappa) V_{*}^{\mu} x(t)^{\top}\left(P_{*} G_{\mathbf{d}}+G_{\mathbf{d}}^{\top} P_{*}\right) x(t)+ \\
x^{\top}(t)\left(P_{*} B \Delta+\Delta^{T} B^{\top} P_{*}\right) x(t)+\sqrt{f^{\top}(t) P_{*} f(t)} .
\end{gathered}
$$

Hence, taking into account $\sigma \in \mathcal{K}$ for sufficiently small $\varepsilon>0$ ( i.e for sufficiently large $t_{N}$ ) we have

$$
x^{\top}(t)\left(P_{*} B \Delta+\Delta^{T} B^{\top} P_{*}\right) x(t) \leq \frac{\rho}{3} V_{*}^{\mu} x(t)^{\top}\left(P_{*} G_{\mathbf{d}}+G_{\mathbf{d}}^{\top} P_{*}\right) x(t) .
$$

Since $\left\|x\left(t_{i}\right)\right\|_{\mathbf{d}} \rightarrow V_{*}$ as $i \rightarrow+\infty$ then for sufficiently small $\varepsilon>0$ (i.e. sufficiently large $t_{N}$ ) the inequality (19) implies

$$
\sqrt{f^{\top}(t) P_{*} f(t)} \leq\left(\frac{\rho}{3}+\kappa\right) V_{*}^{\mu} x(t)^{\top}\left(P_{*} G_{\mathbf{d}}+G_{\mathbf{d}}^{\top} P_{*}\right) x(t) .
$$

Therefore, we have

$$
\dot{V}_{*}(x(t)) \leq-\frac{\rho}{3} V_{*}^{\mu} x(t)^{\top}\left(P_{*} G_{\mathbf{d}}+G_{\mathbf{d}}^{\top} P_{*}\right) x(t)
$$

and the solution of the closed-loop system decays exponential implying the existence of an instant of time $t^{*}>t_{N}$ such that $\left\|x\left(t^{*}\right)\right\|_{\mathbf{d}}<V^{*}$. This contradicts our supposition and means $\lim _{i \rightarrow \infty}\left\|x\left(t_{i}\right)\right\|_{\mathbf{d}}=0$. Hence, taking into account the Lyapunov stability proven above we conclude the global asymptotic stability of the closed-loop system with the switched homogeneous control (28).

The linear switched control (28) is obtained from the non-linear homogeneous one. It can be utilized, for example, in the case when the control system is already equipped with a linear (e.g. analog) controller allowing a dynamic change of feedback gains with some sampling period.

According to the corollary, the proposed sampled-time realization of the implicit homogeneous controller guarantees asymptotic stabilization of the closed-loop system independently of the dwell time (a time between to sampling instants). Such property is rather unusual for sampled and switched control systems with additive disturbances [38]. However, without any assumption on the dwell-time we cannot estimated the convergence rate of this system. Obviously, if the dwell time tends to zero the convergence rate tends to the rate of the original continuous system.

Some advanced schemes for a discrete-time approximation of homogeneous control systems are developed in [39]. They preserve the convergence rate(e.g. finite/fixed time) of the origin continuous-time homogeneous system in its discrete-time counterpart. However, this algorithm still needs on-line computation of the canonical homogeneous norm (or its discrete-time analog). Fortunately, rather simple numerical procedures can be utilized for this purpose.

Let we have some sequence of time instants $0=t_{0}<t_{1}<t_{2}<\ldots$ and $\lim t_{i}=+\infty$. Let $a, b$ be the parameters of the sat function defined in the previous section.

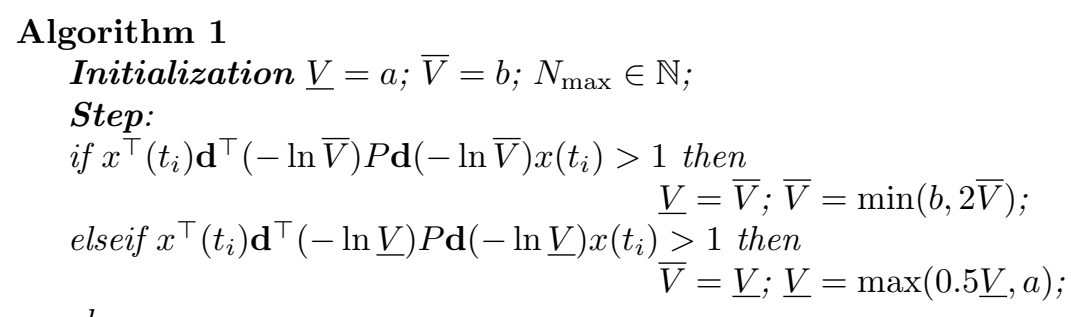

else

$$
\begin{aligned}
& \text { for } i=1: N_{\max } \\
& \quad V=\frac{V+\bar{V}}{2} ; \\
& \quad \text { if } x{ }^{\top}\left(t_{i}\right) \mathbf{d}^{\top}(-\ln \underline{V}) P \mathbf{d}(-\ln \underline{V}) x\left(t_{i}\right)<1 \text { then } \\
& \quad \bar{V}=V ;
\end{aligned}
$$




$$
\begin{aligned}
& \text { else } \underline{V}=V ; \\
& \text { endif; } \\
& \text { endfor; } \\
& \text { endif; } \\
& \left\|x\left(t_{i}\right)\right\|_{\mathbf{d}} \approx \bar{V} ;
\end{aligned}
$$

Let $x\left(t_{i}\right) \in \mathbb{R}^{n} \backslash\{\mathbf{0}\}$ be a given vector and $a=0, b=+\infty$. If the Step of the presented algorithm is applied recurrently many times to the same $x\left(t_{i}\right)$ then Algorithm 1 realizes:

1) a localization of the unique positive root of the equation $\left\|\mathbf{d}(-\ln V) x\left(t_{i}\right)\right\|=1$ with respect to $V>0$, i.e. $V \in[\underline{V}, \bar{V}]$;

2) improvement of the obtained localization by means of the bisection method, i.e. $(\bar{V}-\underline{V}) \rightarrow 0$.

Such an application of Algorithm 1 allows us to calculate $V \approx\left\|x\left(t_{i}\right)\right\|_{\mathbf{d}}$ with rather high precision but it requests a high computational capability of a control device. If the computational power is very restricted, then the Step of Algorithm 1 may be realized just once at each sampled instant of time. Theorem 6 implies practical stability of the closed-loop system in this case. Indeed, Theorem 6 proves that the dhomogeneous ball $\bar{B}_{\mathbf{d}}(\bar{V})$ is a strictly positively invariant set of the the closed-loop system with the control $u(x)=\bar{V}^{\mu} K \mathbf{d}(-\ln \bar{V})$ ). If the root of the equation $\left\|\mathbf{d}(-\ln V) x\left(t_{i}\right)\right\|=0$ is localized (i.e. $\left.x\left(t_{i}\right) \leq \bar{V}\right)$, Algorithm 1 always selects the upper estimate of $V$ to guarantee $x\left(t_{i}\right) \in \bar{B}_{\mathbf{d}}(\bar{V})$. This means that $\left\|x\left(t_{i}\right)\right\|_{\mathbf{d}}$ never leaves the ball $\bar{B}_{\mathbf{d}}(\bar{V})$ even when $x(t)$ varies in time.

The parameters $a$ and $b$ defines lower and upper admissible values for $V$. As explained in the previous section this restrictions are caused by practical issues. For instance, the parameter $a$ can not be selected arbitrary small due to finite numerical precision of digital devices and measurement errors, which may imply $x\left(t_{i}\right) \notin \bar{B}_{\mathbf{d}}(\bar{V})$ due to the computational errors.

\section{Example: Homogenization of a Linear Quadrotor Control}

\subsection{Model Description}

The dynamic model of quadrotor has been well established in the previous research [40, 41]. The dynamic model of quadrotor might be expressed in three different frames: the inertial, horizon and body frame, denoted by $I, H$ and $B$ respectively. Frame $I$ and $B$ are depicted in Fig. 2 (left). The frame $H$ is illustrated in Fig. 2 (right). Precisely, the body frame is attached to the mass center of quadrotor. And the $\phi, \theta, \psi$ are Euler angles defined in the initial frame.

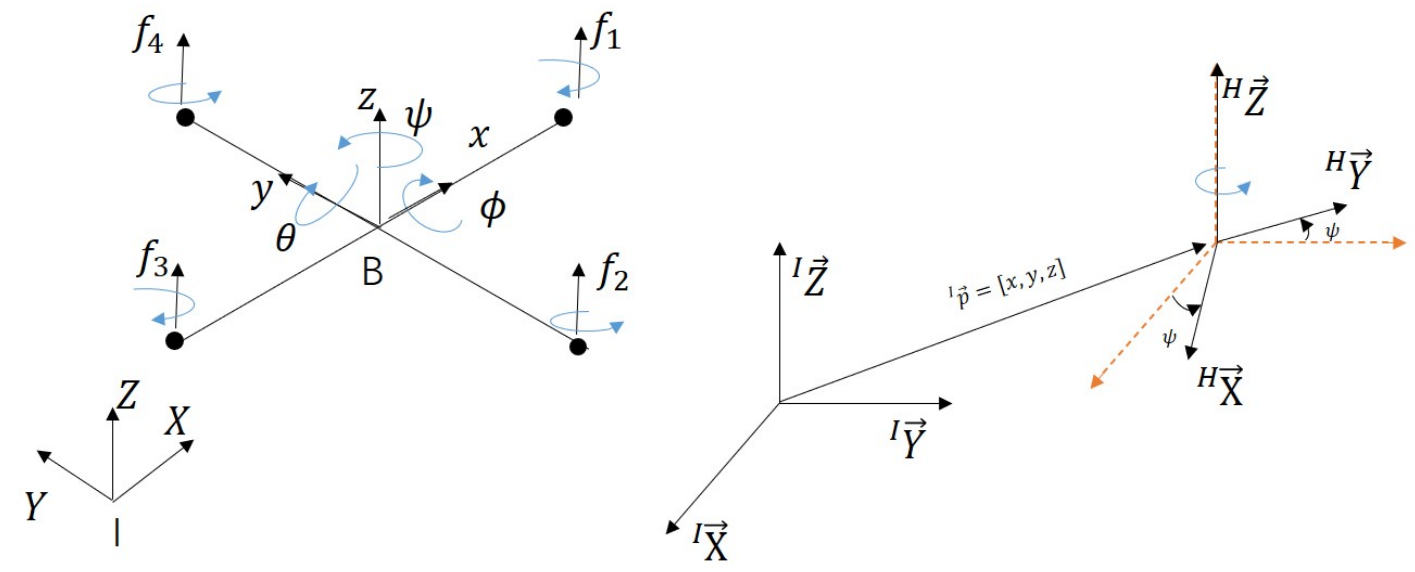

Figure 2: Quadrotor coordinates and the horizon frame

In this paper, the hub forces and moments are negligible [41]. Therefore, the thrust force $\left(F_{T}\right)$ and the gravity $(\mathrm{mg})$ are two main forces to result in the quadrotor's motion. The thrust force is perpendicular to the quadrotor's main body plane (along the body frame $\mathrm{z}$ axis). The linear acceleration of quadrotor 
in different frames can be projected to the horizon frame by using roll $\phi$, pitch $\theta$ and yaw $\psi$, which is ${ }^{H} \vec{a}={ }^{H} R_{B}{ }^{B} \vec{a}-{ }^{H} R_{I}{ }^{I} \vec{g}$, where

$$
{ }^{H} R_{B}=\left[\begin{array}{ccc}
\cos \theta & \sin \theta \sin \phi & \sin \theta \cos \phi \\
0 & \cos \phi & -\sin \phi \\
-\sin \theta & \cos \theta \sin \phi & \cos \theta \cos \phi
\end{array}\right],{ }^{H} R_{I}=\left[\begin{array}{ccc}
\cos \psi & -\sin \psi & 0 \\
\sin \psi & \cos \psi & 0 \\
0 & 0 & 1
\end{array}\right]
$$

Thus

$$
{ }^{H}\left[\begin{array}{c}
\ddot{x} \\
\ddot{y} \\
\ddot{z}
\end{array}\right]=\left[\begin{array}{ccc}
\cos \theta & \sin \theta \sin \phi & \sin \theta \cos \phi \\
0 & \cos \phi & -\sin \phi \\
-\sin \theta & \cos \theta \sin \phi & \cos \theta \cos \phi
\end{array}\right]^{B}\left[\begin{array}{c}
0 \\
0 \\
\frac{F_{T}}{m}
\end{array}\right]-\left[\begin{array}{ccc}
\cos \psi & \sin \psi & 0 \\
-\sin \psi & \cos \psi & 0 \\
0 & 0 & 1
\end{array}\right]^{B}\left[\begin{array}{l}
0 \\
0 \\
g
\end{array}\right]
$$

which yields the following nonlinear model [41]:

$$
{ }^{H} \vec{a}=\left[\begin{array}{c}
\ddot{x} \\
\ddot{y} \\
\ddot{z}
\end{array}\right]=\left[\begin{array}{c}
\frac{F_{T}}{m} \sin \theta \cos \phi \\
-\frac{F_{T}}{m} \sin \phi \\
\frac{F_{T}}{m} \cos \theta \cos \phi-g
\end{array}\right]
$$

where $^{H}\left[\begin{array}{c}\ddot{x} \\ \ddot{y} \\ \ddot{z}\end{array}\right]$ represents the linear accelerations in horizon frame.

According to the Euler equation

$$
J \dot{\omega}+\omega \times J \omega=\tau
$$

where $\omega=L_{i}^{B} \dot{\Theta}, \omega$ is the angle rate projected on the body frame, $J$ is the inertia matrix, $\tau$ is the rotation torque and

$$
\begin{aligned}
\dot{\Theta}=\left[\begin{array}{c}
\dot{\phi} \\
\dot{\theta} \\
\dot{\psi}
\end{array}\right], & L_{i}^{B}=\left[\begin{array}{ccc}
1 & 0 & -\sin \theta \\
0 & \cos \phi & \sin \phi \cos \theta \\
0 & -\sin \phi & \cos \phi \cos \theta
\end{array}\right] \\
\tau=\left[\begin{array}{c}
\tau_{\phi} \\
\tau_{\theta} \\
\tau_{\psi}
\end{array}\right], & J=\left[\begin{array}{ccc}
I_{x x} & 0 & 0 \\
0 & I_{y y} & 0 \\
0 & 0 & I_{z z}
\end{array}\right]
\end{aligned}
$$

where $I_{x x}, I_{y y}$ and $I_{z z}$ are the principle roll, pitch and yaw moments of inertia. $\tau_{\phi}, \tau_{\theta}$ and $\tau_{\psi}$ are the corresponding applied torques in body frame. Then the angular motion equations are

$$
\begin{aligned}
I_{x x} \ddot{\phi} & =\bar{u}_{2} \\
I_{y y} \ddot{\theta} & =\bar{u}_{3} \\
I_{z z} \ddot{\psi} & =\bar{u}_{4}
\end{aligned}
$$

where

$$
\begin{aligned}
& \bar{u}=\left[\begin{array}{l}
\bar{u}_{2} \\
\bar{u}_{3} \\
\bar{u}_{4}
\end{array}\right]=\left[\begin{array}{ccc}
1 & \frac{I_{x x} \sin \phi \tan \theta}{I_{y y}} & \frac{I_{x x} \cos \phi \tan \theta}{I_{z z}} \\
0 & \cos \phi & \frac{-I_{y y} \sin \phi}{I_{z z}} \\
0 & \frac{I_{z z} \sin \phi \sec \theta}{I_{y y}} & \cos \phi \sec \theta
\end{array}\right]\left[\begin{array}{l}
u_{2} \\
u_{3} \\
u_{4}
\end{array}\right]-\left[\begin{array}{ccc}
1 & \frac{I_{x x} \sin \phi \tan \theta}{I_{y y}} & \frac{I_{x x} \cos \phi \tan \theta}{I_{z z}} \\
0 & \cos \phi & \frac{-I_{y y} \sin \phi}{I_{z z}} \\
0 & \frac{I_{z z} \sin \phi \sec \theta}{I_{y y}} & \cos \phi \sec \theta
\end{array}\right]\left[\begin{array}{l}
r_{1} \\
r_{2} \\
r_{3}
\end{array}\right] \\
& {\left[\begin{array}{lll}
u_{2} & u_{3} & u_{4}
\end{array}\right]=\left[\begin{array}{lll}
\tau_{\phi} & \tau_{\theta} & \tau_{\psi}
\end{array}\right]}
\end{aligned}
$$

and

$$
\mathbf{r}=\left(\begin{array}{l}
r_{1} \\
r_{2} \\
r_{3}
\end{array}\right)=J\left(\frac{\partial L_{i}^{B}}{\partial \phi} \dot{\phi}+\frac{\partial L_{i}^{B}}{\partial \theta} \dot{\theta}\right) \dot{\Theta}+L_{i}^{B} \dot{\Theta} \times\left(J L_{i}^{B} \dot{\Theta}\right)
$$

In order to apply the proposed method to design homogeneous PID for the nonlinear quadrotor model (30) and (31), the following will firstly present the linear PID controller for the linearized model of (30) and (31), and finally get the homogeneous PID for the nonlinear model (30) and (31) from the linear PID controller. 


\subsection{Linearized models}

To design homogeneous controllers, let us consider initially linearized model of the system assuming that $\phi$ and $\theta$ are small,

$$
{ }^{H} \vec{a}=\left[\begin{array}{c}
\ddot{x} \\
\ddot{y} \\
\ddot{z}
\end{array}\right]=\left[\begin{array}{c}
\frac{F_{T}}{m} \sin \theta \cos \phi \\
-\frac{F_{T}}{m} \sin \phi \\
\frac{F_{T}}{m} \cos \theta \cos \phi-g
\end{array}\right] \approx\left[\begin{array}{c}
g \theta \\
-g \phi \\
u_{1}
\end{array}\right]
$$

and

$$
\left[\begin{array}{l}
\bar{u}_{2} \\
\bar{u}_{3} \\
\bar{u}_{4}
\end{array}\right] \approx\left[\begin{array}{l}
u_{2} \\
u_{3} \\
u_{4}
\end{array}\right], \quad u_{1}=F_{T}-m g
$$

$\zeta$-subsystem. Denoting $\zeta=(x, y, \dot{x}, \dot{y}, \theta,-\phi, \dot{\theta},-\dot{\phi})^{\top}$ from (4.2) and (31) we derive

$$
\begin{aligned}
\dot{\zeta} & =A_{\zeta} \zeta+B\left(\begin{array}{l}
u_{2} \\
u_{3}
\end{array}\right) \\
\ddot{\psi} & =\frac{u_{4}}{I_{z z}} \\
\ddot{z} & =\frac{u_{1}}{m}
\end{aligned}
$$

where

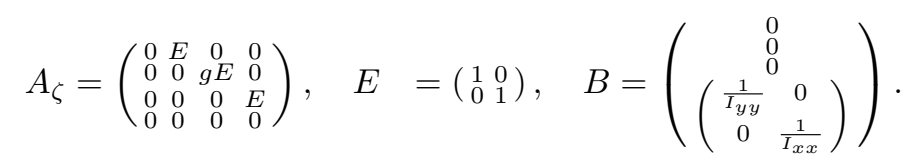

$\psi$-subsystem. Denote $\Psi=\left[\begin{array}{l}\psi \\ \dot{\psi}\end{array}\right]$, then the subsystem (34) becomes

$$
\dot{\Psi}=A_{\psi} \Psi+B_{\psi} u_{4}
$$

where $A_{\psi}=\left[\begin{array}{ll}0 & 1 \\ 0 & 0\end{array}\right], B_{\psi}=\left[\begin{array}{c}0 \\ \frac{1}{I_{z z}}\end{array}\right]$.

$z$-subsystem. Denote $Z=\left[\begin{array}{l}z \\ \dot{z}\end{array}\right]$, then system (35) becomes

$$
\dot{Z}=A_{z} Z+B_{z} u_{1}
$$

where $A_{z}=\left[\begin{array}{ll}0 & 1 \\ 0 & 0\end{array}\right], B_{z}=\left[\begin{array}{c}0 \\ \frac{1}{m}\end{array}\right]$

\subsection{Experimental setup}

In this paper, the test platform is Quanser QDrone ${ }^{T M}$ which is a midsize quadrotor equipped with a powerful onboard Intel Aero Compute Board. The hardware parameters of quadrotor (provided by the manufacturer) are in Table 1. The position and linear velocity are measured by OptiTrack ${ }^{\top \mathrm{T}}$ and computed on board respectively. The angle rate $\omega$ are measured by IMU and the attitude angle are computed from the measurement of IMU.

The PID controller given by the manufacturer has the following form:

$$
u_{1}=K_{z}\left(\begin{array}{c}
z \\
\dot{z}
\end{array}\right)+\int K_{I} z d t, \quad\left(\begin{array}{l}
u_{2} \\
u_{3}
\end{array}\right)=K_{\zeta} \zeta, \quad u_{4}=K_{\psi}\left(\begin{array}{l}
\psi \\
\dot{\psi}
\end{array}\right)
$$

with the parameters

$$
\begin{aligned}
& K_{\psi}=\left[\begin{array}{ll}
-0.59 & 0.11
\end{array}\right] \quad K_{z}=\left[\begin{array}{ll}
-35 & -14
\end{array}\right], K_{I}=-4 \\
& K_{\zeta}=\left(\begin{array}{cccccccc}
-2.91 & 0 & -1.45 & 0 & -1.85 & 0 & -0.16 & 0 \\
0 & -3.53 & 0 & -1.76 & 0 & -2.25 & 0 & -0.20
\end{array}\right) \text {. }
\end{aligned}
$$

We use there gains of linear controller in order to design a homogeneous one. 
Table 1: Quadrotor Model Parameters given by the manufacturer

\begin{tabular}{|c|c|c|c|}
\hline Parameter & Description & Value & Units \\
\hline \hline $\mathrm{g}$ & Gravity & 9.8 & $\mathrm{~m} / \mathrm{s}^{2}$ \\
$\mathrm{~m}$ & Mass & 1.07 & $\mathrm{~kg}$ \\
$L_{\text {roll }}$ & Roll motor distance & 0.2136 & $\mathrm{~m}$ \\
$L_{\text {pitch }}$ & Pitch motor distance & 0.1758 & $\mathrm{~m}$ \\
$I_{x x}$ & Roll Inertia & $6.85 \times 10^{-3}$ & $\mathrm{kgm}^{2}$ \\
$I_{y y}$ & Pitch Inertia & $6.62 \times 10^{-3}$ & $\mathrm{kgm}^{2}$ \\
$I_{z z}$ & Yaw Inertia & $1.29 \times 10^{-2}$ & $\mathrm{kgm}^{2}$ \\
$k$ & Thrust Coefficient & $1.93 \times 10^{-8}$ & $\frac{\mathrm{N}}{R M^{2}}$ \\
$c$ & Drag Coefficient & $0.26 \times 10^{-9}$ & $\frac{\mathrm{Nm}^{2}}{R P M^{2}}$ \\
\hline
\end{tabular}

\subsection{Homogenization of linear controllers}

The pairs $\left\{A_{\zeta}, B_{\zeta}\right\},\left\{A_{z}, B_{z}\right\}$ and $\left\{A_{\psi}, B_{\psi}\right\}$ are controllable, the matrix $A_{\zeta}$ is $\mathbf{d}_{\zeta}$-homogeneous of the degree -1 with $\mathbf{d}_{\zeta}(s)=\operatorname{diag}\left\{e^{4 s} E, e^{3 s} E, e^{2 s} E, e^{s} E\right\}, s \in \mathbb{R}$, the matrix $A_{\phi}$ is $\mathbf{d}_{\phi}$-homogeneous of the degree -1 with $\mathbf{d}_{\phi}(s)=\operatorname{diag}\left\{e^{2 s}, e^{s}\right\}$, and the matrix $A_{z}$ is $\mathbf{d}_{z}$-homogeneous of the degree -0.5 with $\mathbf{d}_{z}(s)=\operatorname{diag}\left\{e^{1.5 s}, e^{s}\right\}$. Moreover, the matrices $A_{\zeta}, A_{\psi}$ and $A_{z}$ are nilpotent. For all subsystems we apply Corollary 2 and derive controllers of the form (15) with $K_{0}=\mathbf{0}$ and the canonical homogeneous norms $\|\zeta\|_{\mathbf{d}_{\zeta}},\|\psi\|_{\mathbf{d}_{\psi}}$ and $\|z\|_{\mathbf{d}_{z}}$ computed using the weighted Euclidean norms with the shape matrices

$$
\begin{aligned}
P_{\zeta} & =\left[\begin{array}{cccccccc}
226.71 & 0 & 81.56 & 0 & 78.43 & 0 & 2.40 & 0 \\
0 & 234.31 & 0 & 84.21 & 0 & 79.33 & 0 & 2.28 \\
81.56 & 0 & 31.32 & 0 & 38.19 & 0 & 12.26 & 0 \\
0 & 84.20 & 0 & 37.47 & 0 & 38.84 & 0 & 1.20 \\
78.43 & 0 & 38.19 & 0 & 59.92 & 0 & 2.53 & 0 \\
0 & 79.33 & 0 & 38.84 & 0 & 62.06 & 0 & 2.40 \\
2.40 & 0 & 1.26 & 0 & 2.53 & 0 & 0.24 & 0 \\
0 & 2.28 & 0 & 1.20 & 0 & 2.40 & 0 & 0.23
\end{array}\right] \\
P_{\psi} & =\left[\begin{array}{cc}
18.41 & 2.19 \\
2.18 & 0.47
\end{array}\right] P_{z}=\left[\begin{array}{cc}
7.86 & 1.21 \\
1.21 & 0.62
\end{array}\right]
\end{aligned}
$$

respectively.

The original linear controller for $z$-subsystem contains the integrator. Taking into account Remark (2) and the form of the dilation $\mathbf{d}_{z}$ we define its homogeneous counterpart as follows

$$
u_{z}(Z)=\|Z\|_{\mathbf{d}_{z}}^{1 / 2} K_{z} \mathbf{d}_{z}\left(-\ln \|Z\|_{\mathbf{d}_{z}}\right) Z+K_{I} \int_{0}^{t} \frac{z(s)}{\|Z(s)\|_{\mathbf{d}_{z}}} d s .
$$

\subsection{Results of experiments}

For practical implementation the term $\|\cdot\|_{\mathbf{d}_{\alpha}}$ in the homogeneous controller of each subsystems has to be replaced (see Section 3 for more details) with $\operatorname{sat}_{a_{\alpha}, b_{\alpha}}\left(\|\cdot\|_{\mathbf{d}_{\alpha}}\right.$ ), where $\alpha \in\{\zeta, \phi, z\}$

The parameters $0<a_{\alpha}<b_{\beta}<+\infty$ has been selected for each subsystem as follows

$$
a_{\zeta}=0.6, a_{\psi}=0.65, a_{z}=0.3, b_{\zeta}=b_{\psi}=b_{z}=1 .
$$

Quanser's linear PID controller and the proposed homogeneous PID controller are compared on the experiment, which consists in the sequential set-points (unit: (m,m,m,rad)) tracking, which are defined as follows:

$$
[x, y, z, \psi]=[0,0,0,0] \rightarrow[0,0,0.4,0] \rightarrow[0.2,0,0.4,0] \rightarrow[0.2,0.2,0.4,0] \rightarrow[0,0,0.4,0] \rightarrow[0,0,0.018,0]
$$



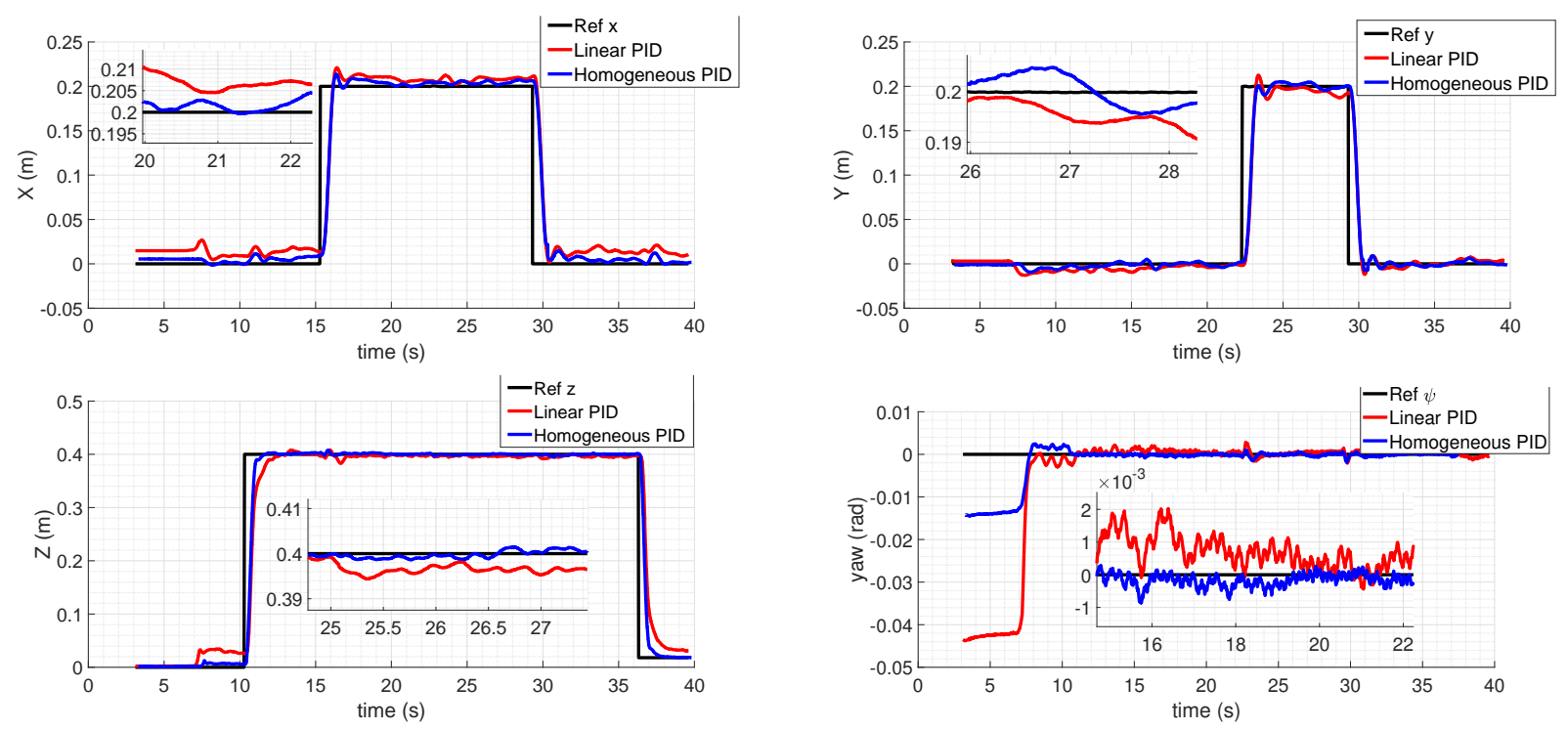

Figure 3: Quadrotor position tracking comparison in $x, y, z$ and $\psi$
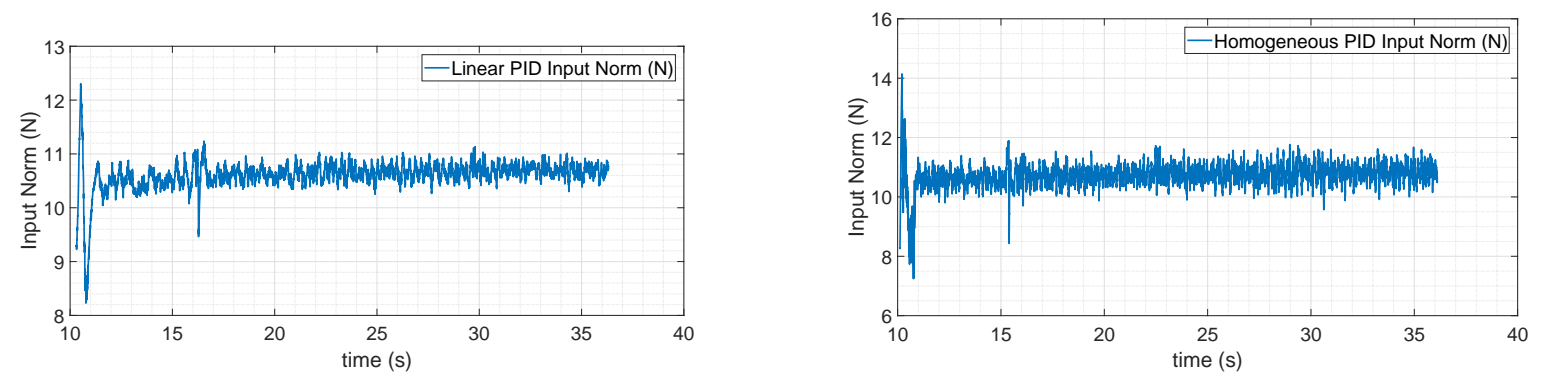

Figure 4: Input $L_{2}$ norm of linear PID signal and Homogeneous PID signal

Fig. 3 depicts the position tracking trajectory of $x, y, z$ and $\psi$ variables, respectively. The homogeneous PID controller has a faster response and a higher precision.

The mean value stabilization errors are compared in Table 2 . They are given by $L_{2}$ norms of the deference between the coordinate and the reference computed in steady states. We define that the steady state starts $\approx 2.5 \mathrm{sec}$ after the set-point assignment and ends at the time instant when the new set-point is assigned. The obtained improvement is more than $40 \%$.

The price of this improvement is a bit more energy consumption, which is estimated by using the $L_{2}$ norm of system inputs. In this test, $L_{2}$ norm of PID controller and homogeneous PID are about 54.14 and 54.75 respectively. The difference between these norms of Quanser PID controller and the homogeneous controller is about $1.1 \%$, i.e., the proposed homogeneous controller consumes only $1.1 \%$ more than the Quanser PID controller, but it can improve about $40 \%$ precision. The input norm of linear PID and Homogeneous PID controller are plotted in Fig. 4.

The robustness of the controllers is also compared by adding a mass $(0.5 \mathrm{~kg})$ for a couple seconds on top of the quadrotor during the flight test. The results of the experiments are depicted in Fig. 5. Both controllers are robust with respect to the load variation. However, the homogeneous controller again demonstrates a better control precision. 
Table 2: Mean values of stabilization errors

\begin{tabular}{|c|c|c|c|}
\hline$L_{2}$ Error $(\mathrm{m})$ & Linear & Homogeneous & Improvement \\
\hline \hline$\|$ error $_{x} \|_{L_{2}}$ & 0.0226 & 0.0127 & $43.8 \%$ \\
\hline$\|$ error $_{y} \|_{L_{2}}$ & 0.0136 & 0.0067 & $50.7 \%$ \\
\hline$\|$ error $_{z} \|_{L_{2}}$ & 0.0203 & 0.0076 & $62.5 \%$ \\
\hline${\text { | } \text { error }_{\psi} \|_{L_{2}}}$ & 0.0043 & 0.0017 & $60.4 \%$ \\
\hline
\end{tabular}
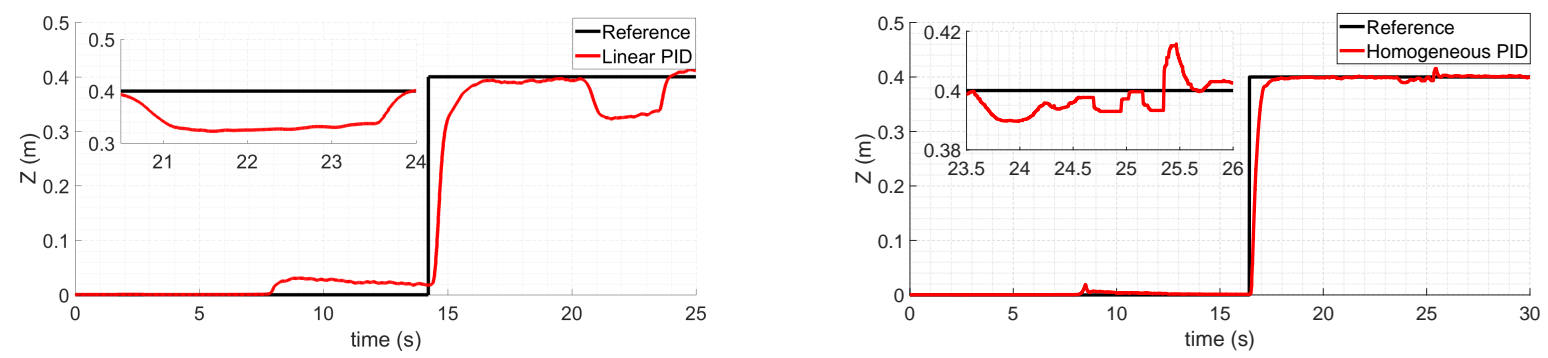

Figure 5: The response of linear controller and homogeneous controller to the added load disturbance

\section{Conclusions}

In this paper, a scheme for an "upgrade" of a linear controller to a non-linear homogeneous one is developed and verified by experiment. The homogeneous controller uses the feedback gain of linear controller and scales it in a generalized homogeneous way which depends on the norm of the system states. The experiments which are tested on the quadrotor platform QDrone of Quanser ${ }^{T M}$ verify the good performance of this controller. The control precision has been improved more than $40 \%$ and the energy consuming increases only about $1.1 \%$. Meanwhile the robustness of controller proposed with respect to the external disturbance is improved as well. It is worth stressing that this method for "homogeneous upgrade" of linear controller can be applied to many other dynamical systems.

\section{Acknowledgments}

The authors acknowledge the support of CSC Grant, CPER DATA and ANR DIGISLID, ANR-18-CE400008. The second author also acknowledges the support of the Government of Russian Federation (Grant 08-08) and the Ministry of Science and Higher Education of Russian Federation, goszadanie no. 20190898. The third author acknowledges the support of the Project of Department of Education of Guangdong Province (No.2019KZDXM037) China, the Project Inventor (I-SITE ULNE, le programme d'Investissements d'Avenir, Métropole Européenne de Lille), and the Project ROBOCOP [ANR-19-CE19-0026].

\section{References}

[1] V.I. Zubov. On systems of ordinary differential equations with generalized homogenous right-hand sides. Izvestia vuzov. Mathematica (in Russian), 1:80-88, 1958.

[2] Y. Orlov. Finite time stability and robust control synthesis of uncertain switched systems. SIAM Journal of Control and Optimization, 43(4):1253-1271, 2005.

[3] A. Polyakov, D. Efimov, E. Fridman, and W. Perruquetti. On homogeneous distributed parameters equations. IEEE Transactions on Automatic Control, 61(11):3657-3662, 2016. 
[4] V. Andrieu, L. Praly, and A. Astolfi. Homogeneous Approximation, Recursive Observer Design, and Output Feedback. SIAM Journal of Control and Optimization, 47(4):1814-1850, 2008.

[5] A. Polyakov. Nonlinear feedback design for fixed-time stabilization of linear control systems. IEEE Transactions on Automatic Control, 57(8):2106-2110, 2012.

[6] Y. Hong. $\mathrm{H} \infty$ control, stabilization, and input-output stability of nonlinear systems with homogeneous properties. Automatica, 37(6):819-829, 2001.

[7] W. Siyuan, A. Polyakov, and G. Zheng. Finite-time LMI based quadrotor control design under time and state constraints. In 2019 IEEE Annual European Control Conference (ECC). IEEE, 2019.

[8] F. Chernous' ko, I. Ananievski, and S. Reshmin. Control of nonlinear dynamical systems: methods and applications. Springer Science \& Business Media, 2008.

[9] A. Levant. Homogeneity approach to high-order sliding mode design. Automatica, 41(5):823-830, 2005.

[10] W. Perruquetti, T. Floquet, and E. Moulay. Finite-time observers: application to secure communication. IEEE Transactions on Automatic Control, 53(1):356-360, 2008.

[11] E. Bernuau, D. Efimov, W. Perruquetti, and A. Polyakov. On homogeneity and its application in sliding mode control. Journal of The Franklin Institute, 351(4):1866-1901, 2014.

[12] J.-M. Coron and L. Praly. Adding an integrator for the stabilization problem. Systems \& Control Letters, 17(2):89-104, 1991.

[13] S. P. Bhat and D. S. Bernstein. Geometric homogeneity with applications to finite-time stability. Mathematics of Control, Signals and Systems, 17:101-127, 2005.

[14] I. Boiko. Non-parametric Tuning og PID Controllers. Springer-Verlag London, 2013.

[15] E.D. Sontag. Nonlinear and Optimal Control Theory, chapter Input to state stability: Basic concepts and results, pages 163-220. Springer-Verlag, Berlin, 2007.

[16] V. I. Utkin. Sliding Modes in Control Optimization. Springer-Verlag, Berlin, 1992.

[17] F. Lopez-Ramirez, A. Polyakov, D. Efimov, and W. Perruquetti. Finite-time and fixed-time observer design: Implicit Lyapunov function approach. Automatica, 87(1):52-60, 2018.

[18] A. Polyakov, D. Efimov, and W. Perruquetti. Robust stabilization of mimo systems in finite/fixed time. International Journal of Robust and Nonlinear Control, 26(1):69-90, 2016.

[19] R. Izmailov. The peak effect in stationary linear-systems with multivariate inputs and outputs. $A u$ tomation and Remote Control, 49(1):40-47, 1988.

[20] A. Polyakov, J.-M. Coron, and L. Rosier. On homogeneous finite-time control for linear evolution equation in Hilbert space. IEEE Transactions on Automatic Control, 63(9):3143-3150, 2018.

[21] Angel Mercado-Uribe, Jaime Moreno, Andrey Polyakov, and Denis Efimov. Integral control design using the implicit lyapunov function approach. Conference on Decision and Control, 2019.

[22] V. V. Khomenuk. On systems of ordinary differential equations with generalized homogenous right-hand sides. Izvestia vuzov. Mathematica (in Russian)., 3(22):157-164, 1961.

[23] M. Kawski. Geometric homogeneity and stabilization. pages 164-169, Lake Tahoe, CA, 1995. Proc. IFAC Nonlinear Control Symposium.

[24] L. Rosier. Etude de quelques problèmes de stabilization. PhD Thesis, Ecole Normale Superieure de Cachan (France), 1993.

[25] A. Pazy. Semigroups of linear operators and applications to partial differential equations, volume 44. Springer Science \& Business Media, 2012. 
[26] A. Polyakov. Sliding mode control design using canonical homogeneous norm. International Journal of Robust and Nonlinear Control, 29(3):682-701, 2018.

[27] D. Efimov and W. Perruquetti. Homogeneity for time-delay systems. IFAC Proceedings Volumes, 44(1):3861-3866, 2011.

[28] A. Bacciotti and L. Rosier. Lyapunov Functions and Stability in Control Theory. Springer, 2001.

[29] L. Rosier. Homogeneous Lyapunov function for homogeneous continuous vector field. Systems $\&$ Control Letters, 19:467-473, 1992.

[30] H. Nakamura, Y. Yamashita, and H. Nishitani. Smooth lyapunov functions for homogeneous differential inclusions. pages 1974-1979. Proceedings of the 41st SICE Annual Conference, 2002.

[31] K. Zimenko, A. Polyakov, D. Efimov, and W. Perruquetti. Robust feedback stabilization of linear MIMO systems using generalized homogenization. IEEE Transactions on Automatic Control, 2020.

[32] W. M. Wonham. Linear Multivariable Control: A Geometric Approach. Springer, 1985.

[33] A. Filippov. Differential equations with discontinuous righthand sides. Springer Science \& Business Media, 1988.

[34] A. Levant. Sliding order and sliding accuracy in sliding mode control. International Journal of Control, 58(6):1247-1263, 1993.

[35] J. La Salle and S. Lefschetz. Stability by Liapunov's Direct Method with Applications. Elsevier Science, 1961.

[36] Y. Orlov. Discontinuous systems: Lyapunov analysis and robust synthesis under uncertainty conditions. Springer, 2008.

[37] A. Polyakov, D. Efimov, and W. Perruquetti. Finite-time and fixed-time stabilization: Implicit Lyapunov function approach. Automatica, 51:332-340, 2015.

[38] D. Liberzon. Switching in systems and control. Springer Science \& Business Media, 2003.

[39] A. Polyakov, D. Efimov, and B. Brogliato. Consistent discretization of finite-time and fixed-time stable systems. SIAM Journal on Control and Optimization, 57(1):78-103, 2019.

[40] S. Bouabdallah. Design and control of quadrotors with application to autonomous flying. Technical report, Epfl, 2007.

[41] S. Bouabdallah and R. Siegwart. Full control of a quadrotor. In 2007 IEEE/RSJ International Conference on Intelligent Robots and Systems, pages 153-158. Ieee, 2007. 